\title{
3D printed bone models in oral and cranio- maxillofacial surgery: a systematic review
}

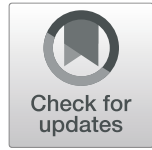

Matteo Meglioli', Adrien Naveau ${ }^{2,3,4}$, Guido Maria Macaluso ${ }^{1,5}$ and Sylvain Catros ${ }^{4,6,7^{*}}$ (D)

\begin{abstract}
Aim: This systematic review aimed to evaluate the use of three-dimensional (3D) printed bone models for training, simulating and/or planning interventions in oral and cranio-maxillofacial surgery.

Materials and methods: A systematic search was conducted using PubMed ${ }^{\oplus}$ and SCOPUS ${ }^{\oplus}$ databases, up to March 10, 2019, by following the Preferred Reporting Items for Systematic reviews and Meta-Analysis (PRISMA) protocol. Study selection, quality assessment (modified Critical Appraisal Skills Program tool) and data extraction were performed by two independent reviewers. All original full papers written in English/French/Italian and dealing with the fabrication of 3D printed models of head bone structures, designed from 3D radiological data were included. Multiple parameters and data were investigated, such as author's purpose, data acquisition systems, printing technologies and materials, accuracy, haptic feedback, variations in treatment time, differences in clinical outcomes, costs, production time and cost-effectiveness.
\end{abstract}

Results: Among the 1157 retrieved abstracts, only 69 met the inclusion criteria. 3D printed bone models were mainly used as training or simulation models for tumor removal, or bone reconstruction. Material jetting printers showed best performance but the highest cost. Stereolithographic, laser sintering and binder jetting printers allowed to create accurate models with adequate haptic feedback. The cheap fused deposition modeling printers exhibited satisfactory results for creating training models.

Conclusion: Patient-specific 3D printed models are known to be useful surgical and educational tools. Faced with the large diversity of software, printing technologies and materials, the clinical team should invest in a 3D printer specifically adapted to the final application.

Keywords: 3D printing, Additive manufacturing, Bone model, Surgical training, Preoperative planning, Simulation

\section{Introduction}

Technological development strongly drives the evolution of oral and cranio-maxillofacial surgery [1]. Among all the additive manufacturing (AM) processes, "three-dimensional printing" (3DP), often used synonymously with additive manufacturing, is playing an ever-growing role. This technology involves the fabrication of objects

\footnotetext{
* Correspondence: sylvain.catros@u-bordeaux.fr

${ }^{4}$ Biotis Laboratory, Inserm U1026, University of Bordeaux, 46 rue Léo-Saignat, 33076 Bordeaux, France

${ }^{6}$ Department of Oral Surgery, UFR d'Odontologie, University of Bordeaux, 46 rue Léo-Saignat, 33076 Bordeaux, France

Full list of author information is available at the end of the article
}

through the deposition of material using a print head, nozzle, or other printing technology [2]. It allows creating objects layer-by-layer through computer-aided design/ computer-aided manufacturing (CAD/CAM). It was originally developed in the 1980s to accelerate the production of small and custom-designed objects, but it revolutionized the prototyping concepts and embraced many applications in manufacturing industries. Later on, AM's applications started to be integrated in several medical techniques and procedures, giving some important inputs to various domains, such as dentistry, maxillofacial surgery, orthopedics and neurosurgery. Frequent clinical applications of 3D printing in everyday practice include 
the fabrication of surgical templates employed to improve the accuracy of the surgery and reduce the duration and morbidity of surgical interventions. It is now applied in routine in oral and craniofacial surgery [3, 4]. More recently, the progress made in the 3D-printing of implantable biomaterials were applied to the fabrication of custom implants, based on patients' radiological data: even if a large amount of these commercial custom implants are milled, 3D-printing technologies can be employed for large bone defects reconstruction like cranioplasty [5] or Temporo-mandibular joint replacement [6].

Three-dimensional printing techniques involve creation of accurate physical 3D models from the patient's radiological data. The first step consists in obtaining the Digital Imaging and COmmunications in Medicine (DICOM) files from patient's imaging exams, such as computed tomography (CT) or magnetic resonance imaging (MRI) scans. Then software is used to transform them into a digital 3D object file, such as standard tessellation language (STL), among other formats. For surgical model fabrication, this new file can be printed with different techniques, such as vat photopolymerization (VP), material extrusion (ME) or binder jetting (BJ). 3D printing encompasses different techniques, each of them having its own benefits and drawbacks (Fig. 1). Several printing materials can be used, each with specific mechanical and accuracy properties. Sometimes, a post-curing step is required to obtain the finished product [7]. The obtained surgical models can fulfill three different purposes: training, planning and simulating. An example of three different models is shown in Fig. 2.

A training model is produced to enhance the quality of the teaching, by allowing students and clinicians to perform high-quality surgical training. Adequate haptic feedback and moderate cost are the most important characteristic of training models. Instead of training on cadaver or animal models, students can enhance their skills on accurate 3DP models that reproduce the haptic feedback of the patient's bone [8]. Although cadaveric specimens have high anatomical and physical validity, they are often challenging to obtain, lack patient-specific pathologic features, and are associated with costs that may be prohibitive to repetitive training [9]. Several authors described temporal bone surgeries [10, 11], implant treatment or maxillary sinus floor augmentation [12] training in realistic in vitro conditions using these 3D-printed models. Thus, one of the most important characteristics of a training model is the low cost.

Secondly, a 3DP model could be made for planning complex surgeries and to fully understand the patient's conditions. The manipulation of the patient anatomical structures helps to get a better understanding of his specific condition and to plan the required surgical interventions [13]. These models are often used in orthognathic

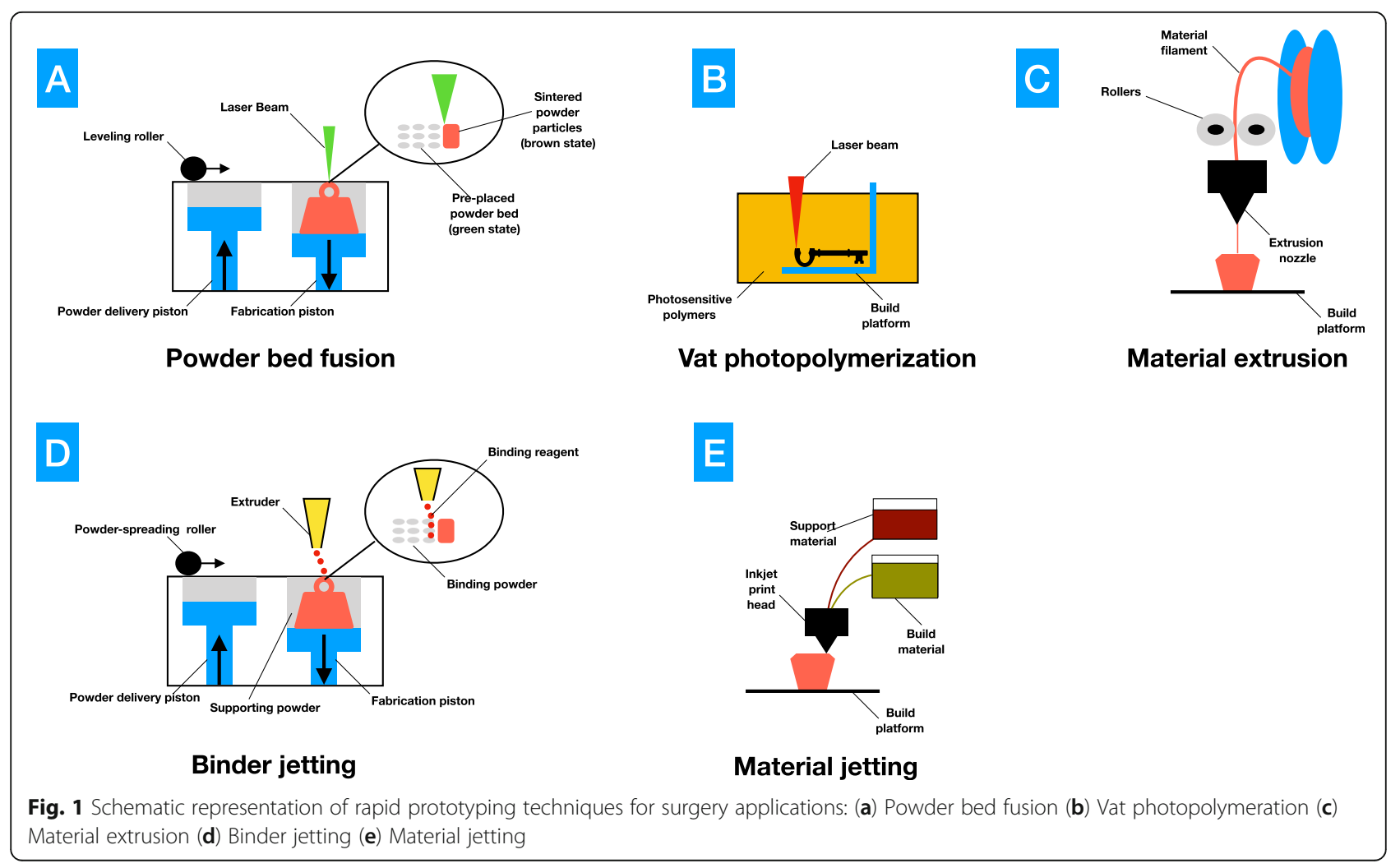




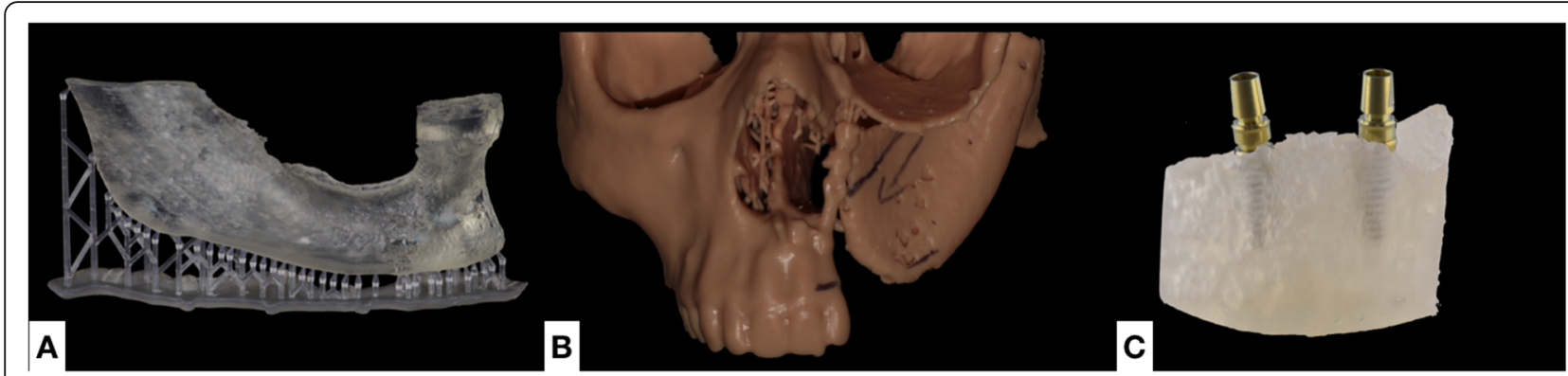

Fig. 2 Examples of anatomical models fabricated by Additive manufacturing. A: A mandible model fabricated using SLA to serve as a template for bone allograft preparation during surgery. B: A model fabricated using SLA to visualize the extent of a bone defect (cleft) for planning the bone reconstructive surgery. C: A model representing a section of a mandible. It was used for training students in dental implant surgery

and reconstructive surgeries, giving the possibility of a better comprehension and more predictable results [14-17]. Measurements and surgical pre-operative evaluations can be performed on these models. Thus, the most important characteristic of a planning model is accuracy.

Finally, a simulating model is produced to perform a surgery before it is done in clinics. This type of models must be accurate with a patient-like haptic feedback. Users can act on these models like they are working on patients. Simulating models are used by clinicians before preforming important surgical interventions, such as tumor excisions [18] and pediatric mastoidectomy [19]. The right mechanical properties, such as the elastic modulus, the stiffness or the drilling force, are fundamental parameters that allow the operator to experience haptic feedback similar to that encountered during actual surgery. Thus, one of the most important characteristic of a simulating model is related to its mechanical properties.

A surgeon wanting to invest in a printer can be overwhelmed in his choice by the numerous options available, between printing materials and technologies. Moreover, the cost-benefit ratio must be taken into consideration, as cheap technologies can be sufficient for some applications. Our hypothesis was that each application of the surgical model could be correlated with a specific 3D printing technology. This systematic review investigated the state of the art of printing materials and techniques proposed to create models for training, simulating and planning interventions in oral and craniofacial surgery. The research focused on the regions of head and neck, scanning articles that belong to different fields: dentistry and oral surgery, maxillofacial surgery, ear-nose-throat surgery and cranial surgery. These results also provided practical suggestions for choosing the optimal 3D printing technique and material for each application objective.

\section{Methods}

\section{Study protocol}

The protocol of this systematic review was registered in 'PROSPERO: International prospective register of systematic reviews' (CRD42019117468) and followed the PRISMA guidelines.

The main question, that was not PICO compliant, was: 'What are the existing printed bone models currently used for training, planning and simulating interventions in oral and cranio-maxillofacial surgery?' The impossibility of using a PICO question and performing a metaanalysis are two missing points of PRISMA checklist.

\section{Search strategy}

Medline (PubMed) database and Scopus database were searched up to March 10, 2019 with the following equation:

(additive manufacturing OR rapid prototyping OR bone model OR bone models OR anatomical models OR anatomical model OR phantom OR phantoms OR simulation model OR simulation models OR 3D -printed models OR $3 D$ printed models OR $3 D$ - printed model OR $3 D$ printed model) AND (planning $O R$ hands-on OR train OR training OR simulation) AND (surgery $O R$ surgical $O R$ dentistry $O R$ dental OR teaching OR pre-operative) AND (maxillofacial OR oral OR skull base OR jaw bones OR jaw OR sinus OR mandible OR temporal bone OR teeth $O R$ maxilla $O R$ human bone OR implant) NOT biology NOT cartilage NOT mathematical

This process only selected articles that had search terms in the title or in the abstract without any restriction on language.

The search was also launched with the following MeSH (PubMed) terms: ("Surgical Procedures, Operative" OR "Teaching") AND "Printing, Three-Dimensional" AND "Models, Anatomic" AND "Head".

Other interesting original research articles were added through manual search.

\section{Science mapping}

A science mapping analysis of subject domains was performed by using keyword co-occurrence networking on 
VOSviewer (free software, version 1.6.15, Centre for Science and Technology Studies, Leiden University, The Netherlands, 2017). A network analysis of the Pubmed $\mathrm{MeSH}$ keywords was generated from the matrix of retrieved papers (threshold value at 90). The MeSH termsdocument matrix allowed to measure document similarities between clusters of topics.

\section{Study selection}

All original full papers written in English/French/Italian and dealing with the fabrication of 3D printed models of head bone structures obtained from 3D imaging data were considered as potentially eligible. Case report, case series, pilot studies and comparative studies were included in this research.

Literature reviews, conference abstracts, articles employing animal tissues or cadaver models or models not aimed to the head region were excluded.

\section{Study analysis}

All the retrieved references, after launching the search algorithm, were managed using Endnote ${ }^{\bullet}$ Abstracts of studies retrieved using the search strategy and those from additional sources were screened independently by three authors (M.M, A.N. and S.C) to identify studies that potentially met the inclusion criteria. Papers fulfilling the inclusion criteria, and those presenting insufficient data in the title and the abstract to make a decision, were selected for full analysis. After reading the full texts, the proper articles were included in an evidence table. Any disagreement over the eligibility of studies was resolved through discussion and consensus among the authors.

\section{Quality assessment}

The quality of the included studies was assessed using a modified version of the Critical Appraisal Skills Programme (CASP) tool [20]. For each of the 10 questions of this tool, there were three possible answers: 'Yes', 'Can't tell' or 'No'. Every 'Yes' scored 1 point, while 'No' or 'Can't tell' scored 0 points. Total scores were converted to percentages and studies were allocated to one of three categories; 'Good quality' for a score of 67$100 \%$, 'Average quality' for $34-66 \%$ and 'Poor quality' for $0-33 \%$.

\section{Data extraction}

The data were extracted and critically appraised by two independent authors (M.M and G.M.M.).

Using a standardized data extraction form, the authors extracted the following data: year of publication, data acquisition system, type of printing technique involved, material, accuracy, production time, haptic feedback, treatment time, clinical outcomes, cost and purpose of the publication: training, planning, simulation (multiple possibilities for each paper).

\section{Data analysis}

A narrative synthesis of the data was conducted due to the heterogeneity of study designs and methods. For the same reason it was not possible to perform a metaanalysis.

In order to address the general question of defining the state of the art of $3 \mathrm{D}$ printing to address the surgical planning, simulation and training needs, the authors identified as crucial these characteristics: accuracy, haptic feedback and cost.

\section{Results}

\section{Search general results}

After database screening and removal of duplicates using Endnote $^{\circ}$ [21], 1157 articles were identified. After abstract screening, 119 studies were selected. After full text reading, 64 articles were selected, plus 5 other articles found among the related ones found by additional manual search (Fig. 3). The main reason for excluding articles was a content not addressing AM models issues. Many excluded articles dealt with analogic models, virtual models or perfusion-based models, or related to the orthopedic and veterinary fields. Six articles were not written in English/French/Italian.

Eight articles were excluded after reviewers' discussion: three authors involved commercial models [22-24], one article dealt with papercraft models [25], one article dealt with a silicon model molded on a 3D printed mold [26] and 3 articles dealt with other surgical fields (orthopedics $[27,28]$ and veterinary sciences [29]).

The spatial representation of the relationships between the Mesh keywords was displayed through a science map (Fig. 4). The networks noticeably highlighted the interest for modelling human patients from imaging data through a computer assisted procedure. Bone surgery, prosthetic rehabilitation and radiotherapy were among the most represented families of clinical applications. "Mandible" was the most co-occurring bone keywords. "Material testing", "treatment outcome" and "clinical competence" were often studied. These graphic references did not show any Mesh keywords regarding the 3D printing technologies themselves (under the threshold).

\section{Quality assessment (Table 1)}

With the modified Critical Appraisals Skills Programme (CASP) tool [20] the reviewers have identified as "high-quality studies" only 8 original researches. The authors classified as "average quality studies" and "poor quality studies" 46 and 15 articles respectively. The lack of quantitative or rigorous tests 


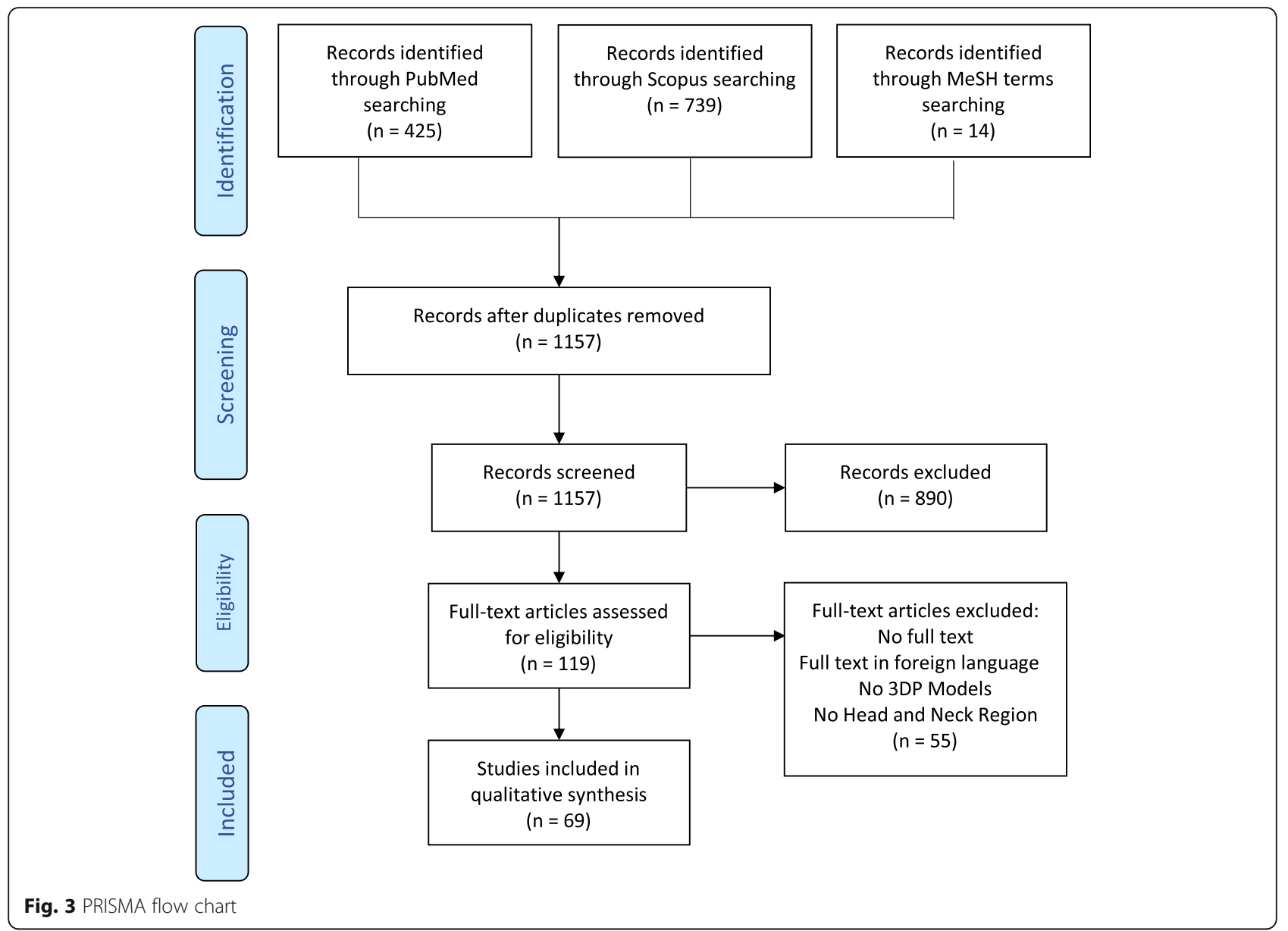

and appropriate study designs were found in most of the selected studies.

\section{General information (Table 2)}

Only 5 articles (6\%) were published before 2008. Ten articles (18\%) were published between 2009 and 2014. Forty-two articles (78\%) were published between 2015 and March 10, 2019.

Among all articles, 3 were pilot studies, 12 were case reports, 4 were case series, 29 were validation studies and only 6 were comparative studies. The authors classified as "validation studies" all original researches that had described and eventually evaluated a process to fabricate a printed bone model. The reviewers classified as "comparative studies" all the researches that compared models printed by different techniques or models printed by different printers using the same technique. Lastly, a paper comparing a bone model to a cadaver's bone was also considered as a comparative study [59].

\section{Purposes of the articles}

The models mentioned in the selected articles were used for different purposes (Table 2). In 31 articles the models were used to plan a surgery, in 19 they were used to simulate the surgery, and in 32 they were used for training of students or clinicians. This total (82) exceeded the total number of papers, as some models were used for multiple purposes.

\section{Surgical field}

The reports on 3DP models concerned multiple surgical domains (Table 2). Oral and maxillofacial surgery had the largest share with $43 \%$ of articles describing the use of AM models, followed by ENT surgery (29\%), dentistry (14\%), and cranial surgery (14\%).

\section{Therapy (Table 2)}

In dentistry, bone models were more frequently used for simulating dental implant placement $[30,41,81]$. In oral and maxillofacial surgery, the models were more frequently used for planning a bone reconstruction [15-17, 


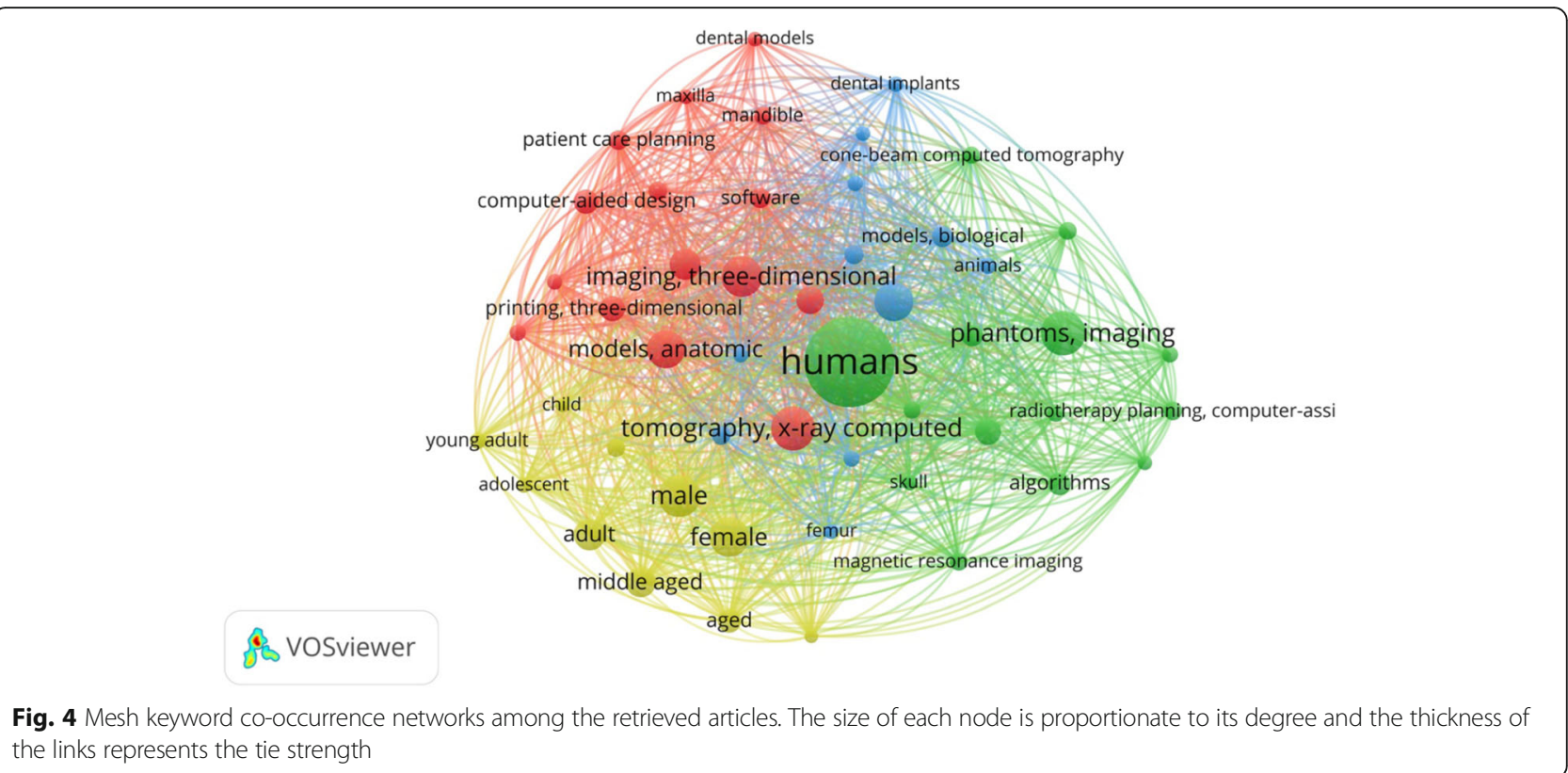

$36,40,47,50,53,72]$ or a tumor removal $[18,49,56]$. The models prepared for ENT surgery were mostly used for training surgical temporal access $[10,31,52,63-68$, 85, 87] and mastoidectomy [39, 51, 59-61]. Finally, in the field of cranial surgery, the models were most frequently used for the training of the pre-sigmoid approach [73] or craniotomy [54].

\section{Image acquisition and processing}

Image acquisition and processing are the first steps to create a 3DP model (Table 3). The most frequently used radiological exam was the $\mathrm{CT}$, followed by cone beam computed tomography (CBCT) and MRI. Software was used to process the radiological data. The most frequently used were Mimics (Materialise, Leuven, Belgium), followed by OsiriX ${ }^{\circ}$ (Pixmeo, Geneva, Switzerland) and 3D Slicer ${ }^{\circ}$ (Surgical Planning Laboratory, Isomics Incorporated, Cambridge, USA). Most of the authors did not specify entirely their digital work-flow to create the STL printable file, making it difficult to reproduce the procedure properly.

\section{Printing systems and materials}

Material extrusion (ME) printing was the preferred technique to create models, followed by material jetting (MJ), respectively $32 \%$ and $22 \%$ of the articles (Table 3). Binder jetting and vat photopolymerization (VP) technique were both involved in $10 \%$ of papers. Powder bed fusion printers were used in $16 \%$ of articles while $9 \%$ did not mentioned the type of 3D printer involved. Among all the references selected, the most frequently used material was acrylonitrile butadiene styrene (ABS), currently only used with material extrusion printers.

\section{Quantitative evaluation}

Accuracy and mechanical characteristics are strongly dependent on the 3D printer's characteristics, on the involved printer material and the size of the model. Only three authors analyzed the mechanical properties of their models through quantitative tests $[43,67,83]$. Due to the differences in the printing materials and measuring methods, it was not possible to compare their results (Table 4). For the same reason, it is impossible to compare the model accuracy, despite the fact that several authors measured the geometric discrepancy (Table 5) [9, $43,51,61,79]$.

\section{Qualitative evaluation}

Most of the authors analyzed the accuracy and haptic feedback of their models using self-made questionnaires and they usually concluded that 3DP models were accurate. Two authors declared respectively less than 100 and $125 \mu \mathrm{m}$ of geometrical discrepancy between the real bone and the model $[33,81]$. Some other authors stated that discrepancies could reach $680 \mu \mathrm{m}$, depending on the size of the model [47]. The haptic feedback was declared adequate in $75 \%$ of articles that investigated it through qualitative tests. A model made of calcium sulfate hemihydrate was considered "too hard" [43] and another 3DP model was "too soft" [65]. Few authors showed that it was difficult to print small bones [10, 52, 63 ] and it was reported that some materials melted during drilling [37, 43]. Few authors reported benefiting of a 
Table 1 CASP questions and results of the quality assessment

\begin{tabular}{ll}
\hline CASP Questions & Results \\
\hline $\begin{array}{l}\text { Was there a clear statement of the aims } \\
\text { of this research? }\end{array}$ & High Quality \\
Is a qualitative methodology appropriate? & 8 Articles \\
$\begin{array}{l}\text { The research was designed appropriate to } \\
\text { address the aims of this research? }\end{array}$ & \\
Was the recruitment strategy appropriate & Average Quality \\
tothe aims of the research? & \\
Was the data collected in a way that adressed & 46 Articles \\
the research issue? & \\
Has the relationship between researcher and & \\
participants been adequately considered? & \\
Have ethical issues been taken into consideration? & Low Quality \\
Was the data analysis sufficiently rigorous? & 15 Articles \\
Is there a clear statement of findings? & \\
How valuable is the research? &
\end{tabular}

reduction in treatment time up to $20 \%$ in the operating room thanks to the models $[18,50,75]$. A third (35\%) of the articles affirmed that the clinical outcomes could potentially be improved by using these techniques, thanks to the better planning and the enhanced comprehension of the patient pathological status. Considering costs and production time, ME printed models were the cheapest $[38,39,42,60]$ and the fastest printers [65, 67]. Costeffectiveness depended on each clinical case and was more striking when several models needed [82]. Only 7\% of the authors suggested that their method was not costeffective $[43,61,63,78]$.

\section{Discussion}

Our original impression was validated by the results, suggesting that, before investing in a printer, the major application of the models needs to be considered. Oral and maxillofacial models for bone surgery applications are mostly used for planning and simulating surgical interventions. Printed products exhibit a wide range of different properties, varying with the machine and the printing material. The main results showed that surgical treatment times can be reduced up to $20 \%$, and that the failure rate tends to decrease $[40,50,71]$. Many authors suggested that clinical outcomes can be improved, but their findings were not supported by any control group $[12,18,19,33-36,41,42,50,56,58,76,78,86]$. Only Banaszewski et al. involved a control group to compare the use of the 3D printed model for planning the surgical reconstruction of the mandible to the traditional technique. They found that the functional and aesthetic results were greater in the group where a 3DP models were applied [41]. A planning model needs to be accurate, but cheap also, as one patient cannot cover extensively all expenses. A training model requires essentially to reproduce relevant haptic feedback and to be an inexpensive investment. These two qualities are also expected to simulate a surgical intervention, but also with a high level of accuracy.

\section{Accuracy}

The MJ printers are currently the most accurate printers, with printed models exhibiting a geometrical discrepancy of $90 \mu \mathrm{m}$ when compared to the patient's bone [76]. The second most accurate printing technique, according to the analyzed articles, is powder bed fusion (PBF). Wanibuchi et al. showed an accuracy ranging from $100 \mu \mathrm{m}$ to $300 \mu \mathrm{m}$ on a temporal bone model measured with a digital caliper [51]. This result was confirmed by another study where a geometrical discrepancy of $150 \mu \mathrm{m}$ was observed between the model and the bone [43]. BJ and ME were reported to be less accurate methods. A geometrical discrepancy of $400 \mu \mathrm{m}$ was observed when using a BJ printer to print a skull base [43]. Most of the researches involving ME printers did not measure quantitatively the models' accuracy, except in one case where they reported a discrepancy reaching $680 \mu \mathrm{m}$ [47]. Our study did not retrieve any paper measuring the accuracy of VP printers, but was previously reported as being high [8].

The lack of quantitative evaluation of the printing accuracy was one of the major limitations of the studies included in this review. The accuracy is related to the printer, the radiological image segmentation process, the size of the printed object and the printing material. For example, a ME printer cannot reach the same precision as an VP or MJ printer due for first to the dimension of the nozzle, but its accuracy could be sufficient to reach the operator's purposes. Depending on the radiological images processing technique a 3D-printed model will always exhibit some discrepancies, the operator has to keep it in mind processing the radiological data.

\section{Haptic feedback}

A good haptic feedback is the most important characteristic of training models and it is strongly dependent on the mechanical characteristics of the printing material. The two fundamental parameters for a model that aims to reproduce the bone haptic feedback are adequate elastic modulus and tensile strength. No quantitative test is currently available to describe the surgeon's haptic feedback during a surgical intervention. Most of the authors created their own questionnaires and asked students and surgeons with different experience about their sensations. Thus, the results were difficult to compare as evaluation protocols were different and also because of the conclusions subjective. However, most of the them were satisfied with their printed models. 
Table 2 General Information of the included studies

\begin{tabular}{|c|c|c|c|c|c|c|}
\hline Year & Author & $\begin{array}{l}\text { Study } \\
\text { Design }\end{array}$ & Country & Domain & Treatment & Target \\
\hline 2019 & Lanis A et al. [30] & Case Report & Chile & Dentistry & Implant treatment & Planning \\
\hline 2019 & Freiser ME et al. [31] & $\begin{array}{l}\text { Validation } \\
\text { Study }\end{array}$ & USA & ENT Surgery & Temporal bone access & $\begin{array}{l}\text { Planning, Simulation, } \\
\text { Training }\end{array}$ \\
\hline 2019 & Zhuo C et al. [32] & $\begin{array}{l}\text { Validation } \\
\text { Study }\end{array}$ & China & ENT Surgery & Endoscopic sinus surgery & Training, Simulation \\
\hline 2018 & Bhadra D et al. [33] & Case Report & India & Dentistry & Endodontic retreatment & Planning \\
\hline 2018 & Lin B et al. [34] & $\begin{array}{l}\text { Validation } \\
\text { Study }\end{array}$ & China & Cranial Surgery & Tumor removal surgery & $\begin{array}{l}\text { Planning, Simulation, } \\
\text { Training }\end{array}$ \\
\hline 2018 & Probst R et al. [10] & $\begin{array}{l}\text { Validation } \\
\text { Study }\end{array}$ & $\begin{array}{l}\text { Switzerland - } \\
\text { Germany }\end{array}$ & Cranial Surgery & $\begin{array}{l}\text { Temporal pediatric surgery, coclear } \\
\text { implantation }\end{array}$ & Training \\
\hline 2018 & Hsieh TY et al. [9] & $\begin{array}{l}\text { Validation } \\
\text { Study }\end{array}$ & USA & ENT Surgery & Endoscopic skull base surgery & $\begin{array}{l}\text { Planning, Simulation, } \\
\text { Training }\end{array}$ \\
\hline 2018 & Reymus M et al. [35] & $\begin{array}{l}\text { Validation } \\
\text { Study }\end{array}$ & Germany & Dentistry & Dental traumatology & Training, \\
\hline 2018 & Sugahara K et al. [36] & Pilot Study & Japan & $\begin{array}{l}\text { Maxillofacial } \\
\text { Surgery }\end{array}$ & Reconstruction and orthognathic surgery & Planning \\
\hline 2018 & Werz SM et al. [37] & $\begin{array}{l}\text { Validation } \\
\text { Study }\end{array}$ & Germany & Dentistry & General dentistry & Training \\
\hline 2018 & Chou PY et al. [16] & $\begin{array}{l}\text { Validation } \\
\text { Study }\end{array}$ & USA & $\begin{array}{l}\text { Maxillofacial } \\
\text { Surgery }\end{array}$ & Cleft lip and palate surgery & Simulation \\
\hline 2018 & Arce K et al. [15] & Case Report & USA & $\begin{array}{l}\text { Maxillofacial } \\
\text { Surgery }\end{array}$ & Mandibular reconstruction & Planning \\
\hline 2018 & Lin WJ et al. [38] & $\begin{array}{l}\text { Validation } \\
\text { Study }\end{array}$ & China & ENT Surgery & Sinus and skull base anatomical study & Training \\
\hline 2018 & Haffner M et al. [39] & $\begin{array}{l}\text { Comparative } \\
\text { Study }\end{array}$ & USA & ENT Surgery & Mastoidectomy & Training \\
\hline 2018 & Jacek B et al. [40] & $\begin{array}{l}\text { Comparative } \\
\text { Study }\end{array}$ & Poland & $\begin{array}{l}\text { Maxillofacial } \\
\text { Surgery }\end{array}$ & $\begin{array}{l}\text { Mandibular reconstruction with bony } \\
\text { free flap }\end{array}$ & Planning \\
\hline 2018 & Alodadi A et al. [41] & Case Report & Saudi & Dentistry & Implantology & Planning \\
\hline 2018 & Reddy GV et al. [42] & $\begin{array}{l}\text { Validation } \\
\text { Study }\end{array}$ & India & $\begin{array}{l}\text { Maxillofacial } \\
\text { Surgery }\end{array}$ & Orthognathic surgery & Training \\
\hline 2017 & Favier V et al. [43] & $\begin{array}{l}\text { Comparative } \\
\text { Study }\end{array}$ & France & Cranial Surgery & Skull base endoscopic surgery & $\begin{array}{l}\text { Planning, Simulation, } \\
\text { Training }\end{array}$ \\
\hline 2017 & Somji SH et al. [12] & Case Report & USA & Dentistry & Sinus augmentation & $\begin{array}{l}\text { Planning, Simulation, } \\
\text { Training }\end{array}$ \\
\hline 2017 & Gargiulo P et al. [19] & Case Report & Iceland & Cranial Surgery & Tumor removal surgery & Planning, Simulation \\
\hline 2017 & $\begin{array}{l}\text { Alrasheed AS et al. } \\
{[44]}\end{array}$ & $\begin{array}{l}\text { Validation } \\
\text { Study }\end{array}$ & Canada & $\begin{array}{l}\text { Maxillofacial } \\
\text { Surgery }\end{array}$ & Endoscopic sinus surgery & Training \\
\hline 2017 & Wang L et al. [45] & $\begin{array}{l}\text { Comparative } \\
\text { Study }\end{array}$ & China & $\begin{array}{l}\text { Maxillofacial } \\
\text { Surgery }\end{array}$ & Aneurysm surgery & $\begin{array}{l}\text { Planning, Simulation, } \\
\text { Training }\end{array}$ \\
\hline 2017 & Javan R et al. [46] & Pilot Study & USA & $\begin{array}{l}\text { Maxillofacial } \\
\text { Surgery }\end{array}$ & Cranial nerve anatomy & Training \\
\hline 2017 & Legocki AT et al. [47] & Case Series & USA & $\begin{array}{l}\text { Maxillofacial } \\
\text { Surgery }\end{array}$ & Craniofacial reconstruction & $\begin{array}{l}\text { Planning, Simulation, } \\
\text { Training }\end{array}$ \\
\hline 2017 & Takahashi K et al. [11] & $\begin{array}{l}\text { Validation } \\
\text { Study }\end{array}$ & Japan & ENT Surgery & Temporal bone dissection & Training \\
\hline 2017 & Yusa K et al. [18] & Case Report & Japan & $\begin{array}{l}\text { Maxillofacial } \\
\text { Surgery }\end{array}$ & Tumor removal & Planning, Simulation \\
\hline 2017 & Ghizoni E et al. [48] & $\begin{array}{l}\text { Validation } \\
\text { Study }\end{array}$ & Brazil & $\begin{array}{l}\text { Maxillofacial } \\
\text { Surgery }\end{array}$ & Craniostenosis & Training \\
\hline 2017 & $\begin{array}{l}\text { Wiedermann JP et al. } \\
\text { [49] }\end{array}$ & Case Report & USA & $\begin{array}{l}\text { Maxillofacial } \\
\text { Surgery }\end{array}$ & Cranio-cervicofacial teratoma & Planning \\
\hline
\end{tabular}


Table 2 General Information of the included studies (Continued)

\begin{tabular}{|c|c|c|c|c|c|c|}
\hline Year & Author & $\begin{array}{l}\text { Study } \\
\text { Design }\end{array}$ & Country & Domain & Treatment & Target \\
\hline 2017 & $\begin{array}{l}\text { Oscar Mario Jacobo et } \\
\text { Al. [50] }\end{array}$ & $\begin{array}{l}\text { Validation } \\
\text { Study }\end{array}$ & Uruguay & $\begin{array}{l}\text { Maxillofacial } \\
\text { Surgery }\end{array}$ & Mandible and orbita recostruction & Planning, Training \\
\hline 2016 & $\begin{array}{l}\text { Wanibuchi M et al. } \\
\text { [51] }\end{array}$ & $\begin{array}{l}\text { Validation } \\
\text { Study }\end{array}$ & Japan & $\begin{array}{l}\text { Maxillofacial } \\
\text { Surgery }\end{array}$ & Mastoidectomy & Training \\
\hline 2016 & Bone TM et al. [52] & $\begin{array}{l}\text { Validation } \\
\text { Study }\end{array}$ & USA & ENT Surgery & Temporal bone surgery & Training \\
\hline 2016 & $\begin{array}{l}\text { Florentino VGB et Al. } \\
\text { [53] }\end{array}$ & Case Report & Brazil & $\begin{array}{l}\text { Maxillofacial } \\
\text { Surger }\end{array}$ & Reconstruction of temporal bone & Planning \\
\hline 2016 & Kondo K et Al. [54] & $\begin{array}{l}\text { Validation } \\
\text { Study }\end{array}$ & Japan & Cranial Surgery & Craniotomy & Training, Simulation \\
\hline 2016 & Lim SH et Al. [55] & $\begin{array}{l}\text { Validation } \\
\text { Study }\end{array}$ & Korea & $\begin{array}{l}\text { Macillo-Facial } \\
\text { Surgery }\end{array}$ & Mandible reconstruction & Planning \\
\hline 2015 & Pacione D et al. [56] & Pilot Study & USA & $\begin{array}{l}\text { Maxillofacial } \\
\text { Surgery }\end{array}$ & $\begin{array}{l}\text { Deformity of the skull base and } \\
\text { craniovertebral junction }\end{array}$ & Planning \\
\hline 2015 & Chan HHL et al. [57] & Case Series & Canada & $\begin{array}{l}\text { Maxillofacial } \\
\text { Surgery }\end{array}$ & Head and neck surgery & Training, Simulation \\
\hline 2015 & Dickinson KJ et al. [58] & Case Report & USA & $\begin{array}{l}\text { Maxillofacial } \\
\text { Surgery }\end{array}$ & Endoscopic resection in esophagus & Planning \\
\hline 2015 & $\begin{array}{l}\text { Hochman JB et al. } \\
\text { [59] }\end{array}$ & $\begin{array}{l}\text { Comparative } \\
\text { Study }\end{array}$ & Canada & $\begin{array}{l}\text { Maxillofacial } \\
\text { Surgery }\end{array}$ & Mastoidectomy and skull base surgery & Training \\
\hline 2015 & Cohen J et al. [60] & $\begin{array}{l}\text { Validation } \\
\text { Study }\end{array}$ & USA & $\begin{array}{l}\text { Maxillofacial } \\
\text { Surgery }\end{array}$ & Mastoidectomy & Training \\
\hline 2015 & Lim C et al. [17] & Case Series & $\begin{array}{l}\text { Australia - New } \\
\text { Zealand }\end{array}$ & $\begin{array}{l}\text { Maxillofacial } \\
\text { Surgery }\end{array}$ & Orbital reconstruction & Planning \\
\hline 2015 & Rose AS et al. [61] & Case Report & USA & ENT Surgery & Mastoidectomy & Planning, Simulation \\
\hline 2015 & Ernoult C. et Al. [62] & Case Series & France & $\begin{array}{l}\text { Maxillofacial } \\
\text { Surgery }\end{array}$ & Reconstructive surgery & Simulation \\
\hline 2015 & Mowry SE. et al. [63] & $\begin{array}{l}\text { Validation } \\
\text { Study }\end{array}$ & USA & ENT Surgery & Temporal bone access & Training \\
\hline 2015 & $\begin{array}{l}\text { Hochman JB et al. } \\
\text { [64] }\end{array}$ & $\begin{array}{l}\text { Validation } \\
\text { Study }\end{array}$ & Canada & ENT Surgery & Temporal bone surgery & Training \\
\hline 2015 & $\begin{array}{l}\text { Longfield EA et al. } \\
\text { [65] }\end{array}$ & $\begin{array}{l}\text { Validation } \\
\text { Study }\end{array}$ & USA & ENT Surgery & Temporal pediatric surgery & Training \\
\hline 2015 & Rose AS et al. [66] & $\begin{array}{l}\text { Validation } \\
\text { Study }\end{array}$ & USA & ENT Surgery & Temporal bone surgery & Training \\
\hline 2014 & $\begin{array}{l}\text { Hochman JB et al. } \\
\text { [67] }\end{array}$ & Case Report & Canada & ENT Surgery & Temporal bone surgery & Training \\
\hline 2014 & $\begin{array}{l}\text { Chenebaux M et al. } \\
\text { [68] }\end{array}$ & $\begin{array}{l}\text { Validation } \\
\text { Study }\end{array}$ & France & ENT Surgery & Temporal bone surgery & Training \\
\hline 2014 & $\begin{array}{l}\text { Narayanan V et al. } \\
\text { [69] }\end{array}$ & $\begin{array}{l}\text { Validation } \\
\text { Study }\end{array}$ & Malaysia & ENT Surgery & Skull base surgery & Training \\
\hline 2014 & Cui J et al. [70] & $\begin{array}{l}\text { Validation } \\
\text { Study }\end{array}$ & China & $\begin{array}{l}\text { Maxillofacial } \\
\text { Surgery }\end{array}$ & Cranial trauma & Planning \\
\hline 2014 & Gil RS et al. [71] & $\begin{array}{l}\text { Validation } \\
\text { Study }\end{array}$ & Spain & $\begin{array}{l}\text { Maxillofacial } \\
\text { Surgery }\end{array}$ & Mandible reconstruction & Planning \\
\hline 2014 & Jardini AL et al. [72] & Case Report & Brasil & Cranial Surgery & Cranial reconstruction & Planning \\
\hline 2013 & Jabbour P et al. [73] & $\begin{array}{l}\text { Validation } \\
\text { Study }\end{array}$ & USA & Cranial Surgery & Presigmoid access & Training \\
\hline 2013 & Li J et al. [74] & Case Series & China & $\begin{array}{l}\text { Maxillofacial } \\
\text { Surgery }\end{array}$ & Orbital reconstruction & Planning \\
\hline 2012 & Ciocca L et al. [75] & Case Report & Italy & $\begin{array}{l}\text { Maxillofacial } \\
\text { Surgery }\end{array}$ & Mandible reconstruction & Planning \\
\hline
\end{tabular}


Table 2 General Information of the included studies (Continued)

\begin{tabular}{|c|c|c|c|c|c|c|}
\hline Year & Author & $\begin{array}{l}\text { Study } \\
\text { Design }\end{array}$ & Country & Domain & Treatment & Target \\
\hline 2011 & Mori K et al. [76] & $\begin{array}{l}\text { Validation } \\
\text { Study }\end{array}$ & Japan & Cranial Surgery & $\begin{array}{l}\text { Cerebral revascularization via skull } \\
\text { approaches }\end{array}$ & Training, Simulation \\
\hline 2011 & Morrison D et al. [77] & Case Report & Australia & Cranial Surgery & Cranial reconstruction & Planning \\
\hline 2010 & Nikzad S et al. [78] & Case Report & Iran & Dentistry & Sinus lift and implant treatment & Planning \\
\hline 2010 & Katatny IE et al. [79] & $\begin{array}{l}\text { Validation } \\
\text { Study }\end{array}$ & Australia & $\begin{array}{l}\text { Maxillofacial } \\
\text { Surgery }\end{array}$ & Mandibular surgery & Planning \\
\hline 2010 & $\begin{array}{l}\text { Lambrecht JTH et al. } \\
\text { [80] }\end{array}$ & Case Series & Switzerland & Dentistry & Oral surgery & Training \\
\hline 2009 & Sohmura T et al. [81] & Case Series & Japan & Dentistry & Implant treatment & Planning, Training \\
\hline 2009 & Mori K et al. [82] & $\begin{array}{l}\text { Validation } \\
\text { Study }\end{array}$ & Japan & ENT Surgery & Skull base surgery & Training \\
\hline 2009 & Radley GJ et al. [83] & Comparative & UK & ENT Surgery & Endoscopic sinus surgery & Training \\
\hline 2009 & Cohen A et al. [84] & Case Series & Syria & $\begin{array}{l}\text { Maxillofacial } \\
\text { Surgery }\end{array}$ & Mandible reconstruction & Planning \\
\hline 2007 & Suzuki M et al. [85] & $\begin{array}{l}\text { Validation } \\
\text { Study }\end{array}$ & Japan & ENT Surgery & Temporal bone surgery & Training \\
\hline 2007 & Mavili ME et al. [86] & Case Series & Turkey & Dentistry & Orthognatic surgery & Planning, Simulation \\
\hline 2004 & Suzuki M et al. [87] & $\begin{array}{l}\text { Validation } \\
\text { Study }\end{array}$ & Japan & ENT Surgery & Temporal bone access & Training \\
\hline 2003 & Muller A et al. [88] & Case Series & Germany & Cranial Surgery & Cranioplasty, tumor removal & Planning, Simulation \\
\hline 1997 & $\begin{array}{l}\text { Löpponen } \mathrm{H} \text { et al. } \\
\text { [89] }\end{array}$ & Case Report & Finland & ENT Surgery & Cochlear implant & Simulation, Training \\
\hline
\end{tabular}

The principal materials for ME printers were polylactic acid (PLA), ABS, polycarbonate (PC), polyethylene terephthalate glycol-modified (PETG) and nylon. In the articles included in this review, there was no consensus regarding the best material to reproduce the bone characteristics. One of the PLA's advantages was its biological properties, as it is known to be biodegradable and non-toxic. Moreover, its haptic feedback was similar to bone at low temperature while drilling [37]. Haffner et al., compared five different materials, and stated that PETG was the most realistic material, followed by PC, PLA and ABS. Nylon properties were considered as not realistic enough [39]. PC was blamed to melt too easily during drilling while ABS could easily reproduce the bone haptic feedback during a cortical mastoidectomy [43]. Favier et al. compared the mechanical characteristics of their models. With Young's Modulus respectively of $2000-3000 \mathrm{~N} / \mathrm{mm} 2$ and $1700 \mathrm{~N} /$ $\mathrm{mm} 2$, the MJ and PBF printed models were considered as realistic. Regarding PBF printing, Mori et al. reported that their model was realistic but the feeling of drilling the cancellous part of the bone model lacked the 'crispy touch' of real bone [82]. This subjective declaration underlines the need for objective criteria to evaluate the haptic feedback of the models. Among all the materials used in the BJ printing technique, cyanoacrylate powder with hydroquinone resembled the most to sheep cortical bone, which was often used as a surgical training model [85].
Unfortunately, no author did quantitative or comparative tests using models printed by VP. However most authors suggested that this technique was efficient for creating adequate models, enhancing the quality of the training $[12,35]$ and suitable for planning complex surgeries [15] or dental implant treatments [30].

The segmentation technique is also an important parameter that it has to be considered for obtaining realistic 3D printed models. Segmenting the trabecular bone structures results in a more realistic haptic feedback when compared to fully solid prints.

\section{Cost}

Cost remains an important parameter that cannot be ignored. There are multiple additional costs in terms of software, printers, printing materials, operators and training hours to produce an in-house 3DP model.

In order to print a model, the first step is to process the DICOM file into an STL file. Among all the commercial software available, the commercial software package Mimics ${ }^{\oplus}$, was the most widely used software, despite a relatively high cost when compared to others. Many free or open source software are available, like ITK-Snap ${ }^{\oplus}$, Slicer ${ }^{\bullet}$ or InVesalius ${ }^{\oplus}[31,35,39,40,60,79]$. OsiriX ${ }^{\oplus}$ possesses a free version (demo) that allows to export STL renderings [12, 46, 47, 52, 63]. 
Table 3 Workflow's analysis of the included studies

\begin{tabular}{|c|c|c|c|c|c|}
\hline Year & Author & $\begin{array}{l}\text { Data } \\
\text { Acquisition }\end{array}$ & $\begin{array}{l}\text { Images Processing } \\
\text { Software }\end{array}$ & Printing Technique & Material \\
\hline 2019 & Lanis A et al. [30] & $\mathrm{CBCT}$ & CoDiagnostiX & Vat photopolymerization & Photosentitive Resin \\
\hline 2019 & $\begin{array}{l}\text { Freiser ME et al. } \\
\text { [31] }\end{array}$ & $\mathrm{CT}$ & 3D Slicer & Vat photopolymerization & Photosentitive Resin \\
\hline 2019 & Zhuo C et al. [32] & $\mathrm{CT}$ & Mimics & Material Extrusion & PLA \\
\hline 2018 & $\begin{array}{l}\text { Bhadra D et al. } \\
\text { [33] }\end{array}$ & $\mathrm{CBCT}$ & - & Material Extrusion & - \\
\hline 2018 & Lin B et al. [34] & $\mathrm{CT}$ & Mimics & Material Jetting & Photosensitive Resin \\
\hline 2018 & $\begin{array}{l}\text { Probst } R \text { et al. } \\
{[10]}\end{array}$ & $\mu \mathrm{CT}$ & - & Binder Jetting & Cast Powder and Bonding Agent \\
\hline 2018 & Hsieh TY et al. [9] & $\mathrm{CT}$ & - & Material Jetting & Photosensitive Resin \\
\hline 2018 & $\begin{array}{l}\text { Reymus } \mathrm{M} \text { et al. } \\
\text { [35] }\end{array}$ & $\mathrm{CBCT}$ & InVesalius & Vat photopolymerization & Photosensitive Resin \\
\hline 2018 & $\begin{array}{l}\text { Sugahara K et al. } \\
\text { [36] }\end{array}$ & $\mathrm{CT}$ & Mimics & Material Jetting & Photosensitive Resin \\
\hline 2018 & $\begin{array}{l}\text { Werz SM et al. } \\
\text { [37] }\end{array}$ & $C T$ & 3D Slicer & Material Extrusion & PLA, ABS \\
\hline 2018 & $\begin{array}{l}\text { Chou PY et al. } \\
\text { [16] }\end{array}$ & $C T$ & Mimics & Material Extrusion & ABS \\
\hline 2018 & Arce K et al. [15] & $\mathrm{CT}$ & Mimics & Vat photopolymerization & Photosentive Resin \\
\hline 2018 & Lin WJ et al. [38] & $C T$ & Mimics & Material Extrusion & PLA \\
\hline 2018 & $\begin{array}{l}\text { Haffner M et al. } \\
\text { [39] }\end{array}$ & $C T$ & Slicer & Material Extrusion & PLA, ABS, Nylon, PETG, PC \\
\hline 2018 & Jacek B et al. [40] & $\mathrm{CT}$ & Slicer & Material Extrusion & ABS \\
\hline 2018 & $\begin{array}{l}\text { Alodadi A et al. } \\
\text { [41] }\end{array}$ & $\mathrm{CBCT}$ & - & - & - \\
\hline 2018 & $\begin{array}{l}\text { Reddy GV et al. } \\
\text { [42] }\end{array}$ & - & - & - & - \\
\hline 2017 & Favier $V$ et al. [43] & CT & $\begin{array}{l}\text { Medical Image } \\
\text { Segmentation Tool }\end{array}$ & $\begin{array}{l}\text { Binder Jetting, Material Jetting, Powder } \\
\text { Bed Fusion, Material Extrusion }\end{array}$ & $\begin{array}{l}\text { Calcium Sulfate Hemihydrate, Opaque } \\
\text { Resin, Polyamide, Polycarbonate }\end{array}$ \\
\hline 2017 & $\begin{array}{l}\text { Somji SH et al. } \\
\text { [12] }\end{array}$ & $\mathrm{CBCT}$ & Osirix & Vat photopolymerization & Photosensitive Resin \\
\hline 2017 & $\begin{array}{l}\text { Gargiulo P et al. } \\
\text { [19] }\end{array}$ & $\mathrm{CT}$ and $\mathrm{MRI}$ & Mimics & Material Extrusion & ABS \\
\hline 2017 & $\begin{array}{l}\text { Alrasheed AS } \\
\text { et al. [44] }\end{array}$ & $C T$ & Mimics & Material Jetting & Photosensitive Resin \\
\hline 2017 & Wang L et al. [45] & CTA & Mimics & Material Jetting & Photosensitive Resin \\
\hline 2017 & Javan R et al. [46] & MRI & Osirix & Powder Bed Fusion & Polyamide \\
\hline 2017 & $\begin{array}{l}\text { Legocki AT et al. } \\
{[47]}\end{array}$ & - & Osirix & Material Extrusion & PLA \\
\hline 2017 & $\begin{array}{l}\text { Takahashi K et al. } \\
\text { [11] }\end{array}$ & CT & ZedView & Binder Jetting & Plastic Powder and Colored Binders \\
\hline 2017 & Yusa K et al. [18] & $\mathrm{CT}$ and $\mathrm{MRI}$ & ZedView & Binder Jetting & Composite Powder \\
\hline 2017 & $\begin{array}{l}\text { Ghizoni E et al. } \\
\text { [48] }\end{array}$ & CT & Mimics & Powder Bed Fusion & Polyamide \\
\hline 2017 & $\begin{array}{l}\text { Wiedermann JP } \\
\text { et al. [49] }\end{array}$ & $\mathrm{CT}$ and $\mathrm{MRI}$ & - & - & - \\
\hline 2017 & $\begin{array}{l}\text { Oscar Mario } \\
\text { Jacobo et Al. [50] }\end{array}$ & CT & - & Material Extrusion & PLA \\
\hline 2016 & $\begin{array}{l}\text { Wanibuchi M } \\
\text { et al. [51] }\end{array}$ & - & - & Powder Bed Fusion & Polyamide and Glass Fiber \\
\hline 2016 & Bone TM et al. & $C T$ & Osirix & Material Extrusion & ABS \\
\hline
\end{tabular}


Table 3 Workflow's analysis of the included studies (Continued)

\begin{tabular}{|c|c|c|c|c|c|}
\hline Year & Author & $\begin{array}{l}\text { Data } \\
\text { Acquisition }\end{array}$ & $\begin{array}{l}\text { Images Processing } \\
\text { Software }\end{array}$ & Printing Technique & Material \\
\hline & [52] & & & & \\
\hline 2016 & $\begin{array}{l}\text { Florentino VGB et } \\
\text { Al. [53] }\end{array}$ & $\mathrm{CT}$ & InVesalius & - & \\
\hline 2016 & $\begin{array}{l}\text { Kondo K et Al. } \\
\text { [54] }\end{array}$ & $\mathrm{CT}$ and MRI & - & Binder Jetting & Calcium sulfate hydrate \\
\hline 2016 & Lim SH et Al. [55] & CT & Mimics & Material Jetting & - \\
\hline 2015 & $\begin{array}{l}\text { Pacione D et al. } \\
{[56]}\end{array}$ & CT & $\begin{array}{l}\text { Philips Intellispace } \\
\text { Portal }\end{array}$ & Material Jetting & Photosensitive Resin \\
\hline 2015 & $\begin{array}{l}\text { Chan HHL et al. } \\
\text { [57] }\end{array}$ & CT & Mimics & Material Extrusion & ABS, ABS and Powder, Polycarbonate \\
\hline 2015 & $\begin{array}{l}\text { Dickinson } \mathrm{KJ} \text { et al. } \\
\text { [58] }\end{array}$ & $\mathrm{CT}$ and MRI & Mimics & Material Jetting & Photosensitive Resin \\
\hline 2015 & $\begin{array}{l}\text { Hochman JB et al. } \\
\text { [59] }\end{array}$ & CT & Mimics & Binder Jetting & Composite Powder \\
\hline 2015 & Cohen J et al. [60] & CT & ITK-Snap & Material Extrusion & ABS \\
\hline 2015 & Lim C et al. [17] & CT & - & Material Extrusion & - \\
\hline 2015 & Rose AS et al. [61] & CT & Mimics & Material Jetting & Photosensitive Resin \\
\hline 2015 & $\begin{array}{l}\text { Ernoult C. et Al. } \\
\text { [62] }\end{array}$ & - & Osirix & Material Extrusion & ABS \\
\hline 2015 & $\begin{array}{l}\text { Mowry SE. et al. } \\
{[63]}\end{array}$ & CT & Osirix & Material Extrusion & ABS \\
\hline 2015 & $\begin{array}{l}\text { Hochman JB et al. } \\
\text { [64] }\end{array}$ & $\mu \mathrm{CT}$ & Mimics & - & - \\
\hline 2015 & $\begin{array}{l}\text { Longfield EA } \\
\text { et al. [65] }\end{array}$ & CT & - & Binder Jetting & - \\
\hline 2015 & Rose AS et al. [66] & CT & Mimics & Material Jetting & Photosensitive Resin \\
\hline 2014 & $\begin{array}{l}\text { Hochman JB et al. } \\
\text { [67] }\end{array}$ & CT & Mimics & Binder Jetting & Composite Powder and Colored Binders \\
\hline 2014 & $\begin{array}{l}\text { Chenebaux M } \\
\text { et al. [68] }\end{array}$ & CT & Magics & Vat photopolymerization & Photosensitive Resin \\
\hline 2014 & $\begin{array}{l}\text { Narayanan } V \text { et al. } \\
\text { [69] }\end{array}$ & $\mathrm{CT}$ and MRI & Biomodroid & Material Jetting & Photosentive Resin \\
\hline 2014 & Cui J et al. [70] & CT & Materialise & Powder Bed Fusion & Polystirene \\
\hline 2014 & Gil RS et al. [71] & CT & Materialise & $\begin{array}{l}\text { Vat photopolymerization, Powder Bed } \\
\text { Fusion }\end{array}$ & - \\
\hline 2014 & $\begin{array}{l}\text { Jardini AL et al. } \\
\text { [72] }\end{array}$ & $\mathrm{CT}$ & InVesalius & Binder Jetting & - \\
\hline 2013 & $\begin{array}{l}\text { Jabbour P et al. } \\
\text { [73] }\end{array}$ & - & - & - & - \\
\hline 2013 & Li J et al. [74] & CT & 3DMSR & Powder Bed Fusion & Polystirene \\
\hline 2012 & $\begin{array}{l}\text { Ciocca L et al. } \\
{[75]}\end{array}$ & $\mathrm{CT}$ & CFT & Material Extrusion & $A B S$ \\
\hline 2011 & Mori K et al. [76] & - & - & Powder Bed Fusion & Polyamide \\
\hline 2011 & $\begin{array}{l}\text { Morrison D et al. } \\
{[77]}\end{array}$ & CT & Mimics & Material Extrusion & ABS \\
\hline 2010 & $\begin{array}{l}\text { Nikzad S et al. } \\
\text { [78] }\end{array}$ & $\mathrm{CT}$ & Simplant & Binder Jetting & Polyamide \\
\hline 2010 & $\begin{array}{l}\text { Katatny IE et al. } \\
\text { [79] }\end{array}$ & $\mathrm{CT}$ & InVesalius & Material Extrusion & $\mathrm{ABS}$ \\
\hline 2010 & $\begin{array}{l}\text { Lambrecht JTH } \\
\text { et al. [80] }\end{array}$ & CBCT & Magics & Material Jetting & - \\
\hline
\end{tabular}


Table 3 Workflow's analysis of the included studies (Continued)

\begin{tabular}{|c|c|c|c|c|c|}
\hline Year & Author & $\begin{array}{l}\text { Data } \\
\text { Acquisition }\end{array}$ & $\begin{array}{l}\text { Images Processing } \\
\text { Software }\end{array}$ & Printing Technique & Material \\
\hline 2009 & $\begin{array}{l}\text { Sohmura T et al. } \\
\text { [81] }\end{array}$ & $\mathrm{CT}$ & VGStudio Max & Material Extrusion & ABS \\
\hline 2009 & Mori K et al. [82] & $\mathrm{CT}$ & - & Powder Bed Fusion & Polyamide \\
\hline 2009 & $\begin{array}{l}\text { Radley GJ et al. } \\
\text { [83] }\end{array}$ & $\mu C T$ & Mimics & Powder Bed Fusion & Polyamide \\
\hline 2009 & $\begin{array}{l}\text { Cohen A et al. } \\
\text { [84] }\end{array}$ & $\mathrm{CT}$ & Magics & Material Jetting & - \\
\hline 2007 & $\begin{array}{l}\text { Suzuki M et al. } \\
\text { [85] }\end{array}$ & $\mathrm{CT}$ & - & Vat photopolymerization & - \\
\hline 2007 & $\begin{array}{l}\text { Mavili ME et al. } \\
\text { [86] }\end{array}$ & $\mathrm{CT}$ & Mimics & Material Jetting & - \\
\hline 2004 & $\begin{array}{l}\text { Suzuki M et al. } \\
\text { [87] }\end{array}$ & $\mathrm{CT}$ & - & Powder Bed Fusion & Polyamide \\
\hline 2003 & $\begin{array}{l}\text { Muller A et al. } \\
{[88]}\end{array}$ & $\mathrm{CT}$ & - & Vat photopolymerization & Photosensitive resin \\
\hline 1997 & $\begin{array}{l}\text { Löpponen } \mathrm{H} \text { et al. } \\
\text { [89] }\end{array}$ & $\mathrm{CT}$ & - & Vat photopolymerization & Photosensitive resin \\
\hline
\end{tabular}

ME printers were the cheapest printers, with reported prices ranging between $2500 \$$ and $3000 \$$ [47, 82]. However, as previously mentioned, these showed limitations in terms of accuracy. PLA and ABS were the cheapest reported materials [32, 37, 39, 47, 52,
82]. PC cost was reported to range from 105\$ to $155 \$$ for the production of a mandible $[43,57]$. The cost for a temporal bone model using BJ was around $400 \$$ [67]. MJ models costs ranged from $270 \$$ to $1000 \$$. One team affirmed that these models were too

Table 4 Quantitative evaluations of 3DP models' mechanical properties

\begin{tabular}{|c|c|c|c|c|}
\hline Year & Author & Objective & Methods & Results \\
\hline 2017 & $\begin{array}{l}\text { Favier V } \\
\text { et al. [43] }\end{array}$ & $\begin{array}{l}\text { Evaluation of several consumer-grade ma- } \\
\text { terials for creating patient-specific 3D- } \\
\text { printed skull base model for anatomical } \\
\text { learning and surgical training. }\end{array}$ & $\begin{array}{l}\text { Four different materials were compared } \\
\text { to fabricate the models } \\
\text { Force sensors were used to evaluate: } \\
\text { - Average force needed to break thin } \\
\text { walls with the surgical suction tip } \\
\text { - Energy spent and reported } \\
\text { instantaneous forces during a } 6 \mathrm{~mm} \\
\text { depth drill }\end{array}$ & $\begin{array}{l}\text { All materials displayed higher mechanical } \\
\text { properties than human cadaver bone } \\
\text { Resin and PA were not adapted because } \\
\text { forces exceeded to break thin walls were } \\
\text { too high ( } 200 \mathrm{~N}) \text {. Using "Multicolor" and } \\
\text { PC, the forces applied were } 1.6 \text { to } 2.5 \text { / } 3.5 \\
\text { times higher than bone. Energy spent } \\
\text { during drilling was respectively } 1.6 \text { and } 2.6 \\
\text { times higher on bone than on PC and } \\
\text { Multicolor. } \\
\text { Finally, PC and Multicolor were the more } \\
\text { adapted materials for this application. }\end{array}$ \\
\hline 2014 & $\begin{array}{l}\text { Hochman } \\
\text { JB et al. } \\
{[67]}\end{array}$ & $\begin{array}{l}\text { To generate a rapid-prototyped temporal } \\
\text { bone model from computed tomography } \\
\text { (CT) data with a specific focus on internal } \\
\text { anatomic fidelity. }\end{array}$ & $\begin{array}{l}\text { Three point bending tests, using a } \\
\text { Texture Analyzer }{ }^{\circledast} \text { were performed to } \\
\text { determine the elastic modulus and yield } \\
\text { point. } \\
\text { Thanks to a 3-axis accelerometer the drill } \\
\text { vibration during the drilling was evalu- } \\
\text { ated on different materials. }\end{array}$ & $\begin{array}{l}\text { The printed bone models were highly } \\
\text { realistic. Void space representation was } \\
\text { excellent with } 88 \% \text { concordance between } \\
\text { cadaveric bone and the resultant rapid- } \\
\text { prototyped temporal bone model. Ultim- } \\
\text { ately, cyanoacrylate with hydroquinone } \\
\text { was determined to be the most appropri- } \\
\text { ate infiltrant for both cortical and trabecu- } \\
\text { lar simulation. The mechanical properties } \\
\text { of all tested infiltrants were similar to real } \\
\text { bone }\end{array}$ \\
\hline 2009 & $\begin{array}{l}\text { Radley GJ } \\
\text { et al. [83] }\end{array}$ & $\begin{array}{l}\text { To fabricate and characterize human sinus } \\
\text { phantoms by } 3 \mathrm{D} \text { printing for surgery } \\
\text { simulation }\end{array}$ & $\begin{array}{l}\text { A modified surgical instrument was used } \\
\text { to evaluate the necessary force to break } \\
\text { thin walls made by test materials } \\
\text { compared to cadaver bone. }\end{array}$ & $\begin{array}{l}\text { The materials that could be successfully } \\
\text { combined into a suitable fluid were } \\
\text { polyurethanes, polishes, and suspended } \\
\text { cellulose/polyesters (hardeners). }\end{array}$ \\
\hline
\end{tabular}


Table 5 Studies including a quantitative evaluation of 3DP models' accuracy

\begin{tabular}{|c|c|c|c|c|}
\hline Year & Author & Objective & Methods & Results / Conclusions \\
\hline 2018 & $\begin{array}{l}\text { Hsieh TY } \\
\text { et al. [9] }\end{array}$ & $\begin{array}{l}\text { Fabrication of sinus and skull base 3D- } \\
\text { printed models for endoscopic skull base } \\
\text { surgery }\end{array}$ & $\begin{array}{l}\text { Numerical measurements and image } \\
\text { navigation were used to localize several } \\
\text { landmarks on the CT images of the } \\
\text { patients compared to the CT of the 3DP } \\
\text { model. } \\
\text { Evaluation of the surgeons perceptions } \\
\text { (Likert scale) after dissecting printed } \\
\text { models (Haptic Feedback and anatomical } \\
\text { accuracy) }\end{array}$ & $\begin{array}{l}\text { Comparisons demonstrated less than } 5 \% \\
\text { difference between the images. } \\
\text { Lickert scores were positive for haptic } \\
\text { feedback }(4,67 / 5) \text { and anatomical } \\
\text { accuracy }(4 / 5)\end{array}$ \\
\hline 2017 & $\begin{array}{l}\text { Favier } V \\
\text { et al. [43] }\end{array}$ & $\begin{array}{l}\text { Evaluation of several consumer-grade ma- } \\
\text { terials for creating patient-specific 3D- } \\
\text { printed skull base model for anatomical } \\
\text { learning and surgical training. }\end{array}$ & $\begin{array}{l}4 \text { different printing materials were } \\
\text { compared for accuracy, surgical forces } \\
\text { needed to break and drill thin walls }\end{array}$ & $\begin{array}{l}\text { PC and PA displayed the highest printing } \\
\text { accuracy. } \\
\text { The use of printed models in PC is a } \\
\text { good substitute to human cadaver bone } \\
\text { for skull base surgery simulation }\end{array}$ \\
\hline 2017 & $\begin{array}{l}\text { Legocki AT } \\
\text { et al. [47] }\end{array}$ & $\begin{array}{l}\text { Evaluation of the feasibility of using low- } \\
\text { cost } 3 D \text { printers for the fabrication of ana- } \\
\text { tomical models for craniofacial } \\
\text { reconstruction }\end{array}$ & $\begin{array}{l}\text { Comparison of in-house printing process } \\
\text { of surgical models vs commercial printed } \\
\text { models. } \\
3 \text { different mandible models } \\
\text { Analogical measurements with digital } \\
\text { caliper + other criteria (cost, production } \\
\text { speed, sterilization ...) }\end{array}$ & $\begin{array}{l}\text { Similar results for the accuracy of both } \\
\text { techniques } \\
\text { Nerve canal visibility, tooth root visibility, } \\
\text { and sterilizability were inferior for in } \\
\text { house models } \\
\text { Overall, the in-house technique is } \\
\text { adapted for education and surgical plan- } \\
\text { ning, including preoperative plates } \\
\text { bending. }\end{array}$ \\
\hline 2016 & $\begin{array}{l}\text { Wanibuchi } \\
\text { M et al. } \\
{[51]}\end{array}$ & $\begin{array}{l}\text { Fabrication of a 3D temporal bone model } \\
\text { and validation of accuracy }\end{array}$ & $\begin{array}{l}\text { Accuracy was investigated by fusion of } \\
\text { the original } C T \text { of patient's temporal bone } \\
\text { and the 3DP model's } C T\end{array}$ & $\begin{array}{l}\text { The differences between both } \mathrm{CT} \text { images } \\
\text { were below } 1 \mathrm{~mm} \\
\text { The printed models are adapted for } \\
\text { surgical training. }\end{array}$ \\
\hline 2015 & $\begin{array}{l}\text { Rose AS } \\
\text { et al. [61] }\end{array}$ & $\begin{array}{l}\text { Producing a patient-specific model for } \\
\text { pre-operative simulation in pediatric oto- } \\
\text { logic surgery }\end{array}$ & $\begin{array}{l}\text { Case report of cholesteatoma } \\
\text { Measurement and comparison of } \\
\text { distances between several anatomic } \\
\text { landmarks (CT scan / Printed model / } \\
\text { During surgery) }\end{array}$ & $\begin{array}{l}\text { The variability was minimal, in terms of } \\
\text { absolute distance (mm) and relative } \\
\text { distance (\%), in measurements between } \\
\text { anatomic landmarks obtained from the } \\
\text { patient intra-operatively, the pre- } \\
\text { operative CT scan and the 3D-printed } \\
\text { models. }\end{array}$ \\
\hline 2010 & $\begin{array}{l}\text { Katatny IE } \\
\text { et al. [79] }\end{array}$ & $\begin{array}{l}\text { Simulation of shape and } C T \text { values of } \\
\text { pulmonary parenchyma and lesions of } \\
\text { various sizes using } 3 D P\end{array}$ & $\begin{array}{l}\text { Comparison of patient original } \mathrm{CT} \text { and } \\
\text { printed model } \mathrm{CT}\end{array}$ & $\begin{array}{l}\text { High accuracy was observed } \\
\text { Patient-specific CT imaging phantoms } \\
\text { can be obtained by FDM printer } \\
\text { It can be used for the calibration of CT } \\
\text { intensity and validation of image } \\
\text { quantification software. }\end{array}$ \\
\hline
\end{tabular}

expensive [66], but two others suggested that they were satisfied by their investment in these models $[15,56]$. LS printers are not cheap, but no authors talked mentioned any price. Printing a skull base using polyamide with an LS printer was reported to cost 250-280\$ [85]. Two teams used VP printers and they declared being satisfied by the results [12, 35]. A forgotten cost is related to the post-curing machine for object printed trough VP technologies.

The production time can also be considered as a decisive cost. ME printers were the fastest, producing a pediatric temporal bone model in $4.5 \mathrm{~h}$ and a mandible with a maxilla in $6 \mathrm{~h}[37,39]$. The production time of an MJ model was less than a day $[9,56]$. The time needed to design the STL file is also important to consider. Only one author discussed about the total production time of a LS printer, and reported a need for 4 to 5 days [43].
The learning curve of a few months to master and to properly use the software for STL processing is obvious. In every case, the conversion from the DICOM to an adequate STL file could take several hours. One author reported that "the 3DP technique is really cost-effective, only if the operator plans to produce several models to amortize the cost of the 3D printer" [82].

It's important to know these costs because the costbenefit ratio has to be considered before investing in this technique that can get several advantages but it presents some drawbacks in comparison to traditional techniques $[63,78]$.

\section{Suggestions}

As pre-surgical tools, 3D models can make the surgical outcomes more predictable and safer, reducing the surgeon's stress and the intervention time $[40,50]$. 


\begin{tabular}{|c|c|c|c|c|}
\hline ME & VAT P & PBF & BJ & $M J$ \\
\hline Accuracy & Accuracy & Accuracy & Accuracy & Accuracy \\
\hline $\begin{array}{l}\text { Haptic } \\
\text { Feedback }\end{array}$ & $\begin{array}{c}\text { Haptic } \\
\text { Feedback }\end{array}$ & $\begin{array}{c}\text { Haptic } \\
\text { Feedback }\end{array}$ & $\begin{array}{c}\text { Haptic } \\
\text { Feedback }\end{array}$ & $\begin{array}{c}\text { Haptic } \\
\text { Feedback }\end{array}$ \\
\hline $\begin{array}{c}\text { Production } \\
\text { Time }\end{array}$ & $\begin{array}{l}\text { Production } \\
\text { Time } \\
\end{array}$ & $\begin{array}{c}\text { Production } \\
\text { Time } \\
\end{array}$ & $\begin{array}{c}\text { Production } \\
\text { Time } \\
\end{array}$ & $\begin{array}{c}\text { Production } \\
\text { Time } \\
\end{array}$ \\
\hline Cost & Cost & Cost & Cost & Cost \\
\hline TRAINING & $\begin{array}{c}\text { TRAINING, } \\
\text { SIMULATING } \\
\text { AND PLANNING }\end{array}$ & PLANNING & PLANNING & $\begin{array}{c}\text { TRAINING, } \\
\text { SIMULATING } \\
\text { AND PLANNING }\end{array}$ \\
\hline
\end{tabular}

Fig. 5 Advantages (green) and limitations (red) of different 3D printing technology to create 3D anatomical models. These characteristics lead the choice for the best 3D printer technique for every clinical or academical purpose

Furthermore, they can strongly improve the quality of clinical education, allowing students to simulate various surgical interventions and to discuss easily about their clinical cases with their mentors [34, 35, 42].

Evaluating the best image processing workflow remains difficult as no author described neither the entire workflow not the human cost involved. The most used training models are made with ME printers. This technology is the cheapest and allows producing suitable training models, despite their limited accuracy. The most appropriate ME printing materials are ABS, PLA and PETG [43]. Temporal bone models printed with PETG were reported to ensure adequate haptic feedback whileperfor drilling, and they were very helpful during training sessions [39].

To obtain an adequate simulation model, BJ and VP printers seem more indicated. They showed adequate performances in reproducing training models of the maxilla and their accuracy is really satisfying for creating planning models [12, 18]. It is also possible to create models for simulating surgeries, thanks to their good haptic feedback $[12,14,18]$. Also MJ printers allow to produce models that provide good haptic feedback and that can be used to simulate complex surgeries before entering the operating room [14]. Generally, they cost more than the VP ones, as well as the $\mathrm{BJ}$ ones $[35,61]$. PBF printers allow to produce accurate bone models in polyamide and glass fiber, but without satisfying haptic feedback [82].

Printing time and cost are very variable, depending directly on the type of 3D printer, the printing material, the accuracy and the mechanical characteristics required. Figure 5 demonstrates the main differences among the analyzed 3D printing technologies, depending on the applications.

\section{Conclusions}

The present literature review showed that nowadays, AM models are useful tools in the surgical field.

Several parameters must be considered before choosing a 3D printing model workflow, such as the processing software, the type of 3D printer, the expected mechanical characteristics, accuracy and haptic feedback of the printing material, the production time and the human and material costs.

Due to the large amount of different parameters that has to be considered by the operator, the financial investment in a $3 \mathrm{D}$ printer should be made with the precise idea of the final application.

\section{Limitations}

This analysis was at first limited by the diversity of workflows and applications, involving different materials, printers and testing methods. Despite difficulties for comparing results from a study to another, some common protocols were found for the 3 main purposes of 3D-printed bone models (planning, simulation and training).

The lack of common reliable qualitative tests to evaluate the models was an evident limitation, thus future studies should focus on standardized methods to evaluate 3D-printed models of bone macro- and microstructures.

\section{Abbreviations}

3D: Three-dimensional; 3DP: Three-dimensional printed; Three-dimensional printing; ABS: Acrylonitrile butadiene styrene; AM: Additive manufacturing; BJ: Binder jetting; CAD/CAM: Computer-aided design/computer-aided manufacturing; CBCT: Cone beam computed tomography; $\mathrm{CT}$ : Computed tomography; DICOM: Digital imaging and communications in medicine; ENT: Ear, nose and throat; ME: Material extrusion; MJ: Material jetting; MRI: Magnetic resonance imaging; PBF: Powder bed fusion; 
PC: Polycarbonate; PETG: Polyethylene terephthalate glycol-modified; PLA: Polylactic acid; STL: Standard triangulation language; VP: Vat photopolymerization

\section{Acknowledgements}

None.

\section{Authors' contributions}

All the authors contributed equally to this manuscript. They worked together to design this study, to analyze the data and to write the manuscript. All authors read and approved the final manuscript.

\section{Funding}

This work was funded by "Agence Nationale de la Recherche" (ANR-16-CE180009-01).

\section{Availability of data and materials}

All data generated or analyzed during this study are included in this published article [and its supplementary information files].

\section{Ethics approval and consent to participate}

Not applicable.

\section{Consent for publication}

Not applicable.

\section{Competing interests}

The authors declare that they have no competing interests.

\section{Author details}

'University Center of Dentistry, Department of Medicine and Surgery, University of Parma, Via Gramsci 14, 43126 Parma, Italy. ${ }^{2}$ Department of Prosthodontics, Dental Science Faculty, University of Bordeaux, 46 rue Léo-Saignat, 33076 Bordeaux, France. ${ }^{3}$ Dental and Periodontal Rehabilitation Unit, Saint Andre Hospital, Bordeaux University Hospital, 46 rue Léo-Saignat, 33076 Bordeaux, France. ${ }^{4}$ Biotis Laboratory, Inserm U1026, University of Bordeaux, 46 rue Léo-Saignat, 33076 Bordeaux, France. ${ }^{5}$ IMEM-CNR, Parco Area delle Scienze 37/A, 43124 Parma, Italy. ${ }^{6}$ Department of Oral Surgery, UFR d'Odontologie, University of Bordeaux, 46 rue Léo-Saignat, 33076 Bordeaux, France. ${ }^{7}$ Service de Chirurgie Orale, CHU de Bordeaux, 46 rue Léo-Saignat, 33076 Bordeaux, France.

\section{Received: 14 April 2020 Accepted: 18 September 2020}

Published online: 20 October 2020

\section{References}

1. Naveau A, Bou C, Sharma A. Evolution of Topics in Maxillofacial Prosthetic Publications. Int J Prosthodont. 2018;31(6):565-8. https://doi.org/10.11607/ ijp.5894.

2. ASTM International. Standard Terminology for Additive Manufacturing Technologies 1,2. 2013:2-4. doi:https://doi.org/10.1520/F2792-12A.2.

3. D'Haese J, Ackhurst J, Wismejier D, et al. Current state of the art of computer-guided implant surgery. Periodontology 2000. 2017;73(25):121-33. https://doi.org/10.1111/prd.12175.

4. Lin HH, Lonic D, Lo LJ, et al. 3D printing in orthognathic surgery - a literature review. J Formos Med Assoc. 2018;117(7):547-58. https://doi.org/ 10.1016/j.jma.2018.01.008

5. Gonzalez-perez-somarriba B, Centeno G, Vallellano C et al.. Evaluation of total alloplastic temporo-mandibular joint replacement with two different types of prostheses : A three-year prospective study. 2016;21(6). doi:https:// doi.org/10.4317/medoral.21189.

6. Charles Champeaux, Sebastien Froelich and Caudron Y. Titanium ThreeDimensional Printed Cranioplasty for Fronto-Nasal Bone Defect 2019:30(6): 1802-1805. doi:https://doi.org/10.1097/SCS.0000000000005493.

7. Park JY, Gao G, Jang J, Cho D. 3D printed structures for delivery of biomolecules and cells: tissue repair and regeneration. J Mater Chem B. 2016. https://doi.org/10.1039/C6TB01662F.

8. Crafts TD, Ellsperman SE, Wannemuehler TJ, Bellicchi TD, Shipchandler TZ, Mantravadi AV. Three-Dimensional Printing and Its Applications in Otorhinolaryngology. Head Neck Surg. 2017;156(6):999-1010. https://doi. org/10.1177/0194599816678372.
9. Hsieh TY, Cervenka B, Dedhia R, Strong EB, Steele T. Assessment of a patient-specific, 3-dimensionally printed endoscopic sinus and skull base surgical model. JAMA Otolaryngol - Head Neck Surg. 2018;144(7):574-9. https://doi.org/10.1001/jamaoto.2018.0473.

10. Probst R, Stump R, Mokosch M, Röösli C. Evaluation of an infant temporalBone model as training tool. Otol Neurotol. 2018;39(6):e448-52. https://doi. org/10.1097/MAO.0000000000001839.

11. Takahashi K, Morita Y, Ohshima S, Izumi S, Kubota Y, Yamamoto Y, Takahashi S, Horii A. Creating an optimal 3D printed model for temporal Bone dissection training. Ann Otol Rhinol Laryngol. 2017;126(7):530-6. https://doi. org/10.1177/0003489417705395.

12. Somji SH, Valladares A, Ho Kim S, Cheng Paul Yu Y, Froum SJ. The use of 3D models to improve sinus augmentation outcomes - a case report. Singap Dent J. 2017;38:63-70. https://doi.org/10.1016/j.sdj.2017.10.002.

13. Marconi S, Pugliese $L$, Botti $M$, et al. Value of $3 D$ printing for the comprehension of surgical anatomy. Surg Endosc. 2017. https://doi.org/10. 1007/s00464-017-5457-5.

14. Xiao Y, Sun X, Wang L, Zhang Y, Chen K, Wu G. The application of 3D printing Technology for Simultaneous Orthognathic Surgery and Mandibular Contour Osteoplasty in the treatment of craniofacial deformities. Aesthet Plast Surg. 2017;41(6):1413-24. https://doi.org/10.1007/s00266-0170914-z.

15. Arce K, Waris S, Alexander AE, Ettinger KS. Novel patient-specific 3D printed fixation tray for mandibular reconstruction with fibular free flaps. J Oral Maxillofac Surg. 2018. https://doi.org/10.1016/j.joms.2018.04.028.This.

16. Chou PY, Hallac RR, Shih E, et al. 3D-printed models of cleft lip and palate for surgical training and patient education. Cleft Palate-Craniofacial J. 2018; 55(3):323-7. https://doi.org/10.1177/1055665617738998.

17. Lim C, Campbell D, Cook N, Erasmus J. A case series of rapid prototyping and intraoperative imaging in orbital reconstruction. Craniomaxillofacial Trauma Reconstr. 2015;08(02):105-10. https://doi.org/10.1055/s-00341395384.

18. Yusa K, Yamanochi H, Takagi A, lino M. Three-dimensional printing model as a tool to assist in surgery for large mandibular tumour: a case report. J Oral Maxillofac Res. 2017;8(2):1-7. https://doi.org/10.5037/jomr.2017.8204.

19. Gargiulo P, Arnadottir I, Gislason M, Edmunds K, Olafsson I. New directions in 3D medical modeling: 3D-printing anatomy and functions in neurosurgical planning. J Healthc Eng. 2017;2017. https://doi.org/10.1155/ 2017/1439643/.

20. Critical Appraisal Skills Programme (2018). CASP Qualitative Checklist. [online] Available at: https://casp-uk.net/wp-content/uploads/2018/01/CASPQualitative-Checklist-2018.pdf. Accessed: 20/11/2018. 1994;(2018).

21. EndNote X9, Clarivate Analytics, Web of Science. Philadelphia; Clarivate. 2013(64).

22. He LH, Purton D, Swain M. A novel polymer infiltrated ceramic for dental simulation. J Mater Sci Mater Med. 2011;22(7):1639-43. https://doi.org/10. 1007/s10856-011-4350-3.

23. He LH, Foster Page L, Purton D. An evaluation of dental operative simulation materials. Dent Mater J. 2012;31(4):645-9. https://doi.org/10.4012/ dmj.2011-264.

24. Güth JF, Ponn A, Mast G, Gernet W, Edelhoff D. Description and evaluation of a new approach on pre-clinical implant dentistry education based on an innovative simulation model. Eur J Dent Educ. 2010;14(4):221-6. https://doi. org/10.1111/j.1600-0579.2009.00614.x.

25. Hiraumi $H_{\text {, Sato } H}$, Ito J. Papercraft temporal bone in the first step of anatomy education. Auris Nasus Larynx. 2017;44(3):277-81. https://doi.org/ 10.1016/j.anl.2016.07.017.

26. Chang DR, Lin RP, Bowe $S$, et al. Fabrication and validation of a low-cost, medium-fidelity silicone injection molded endoscopic sinus surgery simulation model. Laryngoscope. 2017;127(4):781-6. https://doi.org/10.1002/ lary.26370.

27. Wang MY, Wang JQ, Shi Y, et al. Printed three-dimensional anatomic templates for virtual preoperative planning before reconstruction of old pelvic injuries. Chin Med J. 2015:128(4):477-82. https://doi.org/10.4103/0366-6999.151088.

28. Brouwers L, Teutelink A, van Tilborg FAJB, de Jongh MAC, Lansink KWW Bemelman M. Validation study of 3D-printed anatomical models using 2 PLA printers for preoperative planning in trauma surgery, a human cadaver study. Eur J Trauma Emerg Surg. 2018;0(0):1-8. https://doi.org/10.1007/ s00068-018-0970-3.

29. Athanasiou KA, Verstraete FJM, Winer JN, Cissell DD, Arzi B, Lucero S. The application of 3-dimensional printing for preoperative planning in oral and 
maxillofacial surgery in dogs and cats. Vet Surg. 2017:46(7):942-51. https:// doi.org/10.1111/vsu.12683.

30. Lanis A, Alvarez del Canto O, Barriga P, Polido WD, Morton D. Computerguided implant surgery and full-arch immediate loading with prefabricatedmetal framework-provisional prosthesis created from a 3D printed model. J Esthet Restor Dent. 2019;31(3):199-208. https://doi.org/10.1111/jerd.12458.

31. Freiser ME, Ghodadra A, Hirsch BE, AA MC. Evaluation of 3D Printed Temporal Bone Models in Preparation for Middle Cranial Fossa Surgery. Otol Neurotol. 2019;40(2):246-53. https://doi.org/10.1097/MAO 0000000000002073 .

32. Zhuo C, Lei L, Yulin Z, et al. Creation and validation of three-dimensional printed models for basic nasal endoscopic training. Int Forum Allergy Rhinol. 2019;00(0):1-7. doi:https://doi.org/10.1002/alr.22306.

33. Bhadra D, Shah NC, Arora A, Meetkumar SD. Deducing a surgical dilemma using a novel three Dimensionaldimensional printing technique Dhaval. J Conserv Dent. 2018;21:582-5. https://doi.org/10.4103/JCD.JCD.

34. Lin B, Zhu Y, Liu Y, et al. Using three-dimensional printing to create individualized cranial nerve models for Skull Base tumor surgery. World Neurosurg. 2018;120:e142-52. https://doi.org/10.1016/..wneu.2018.07.236

35. Reymus M, Fotiadou C, Hickel R, Diegritz C. 3D-printed model for hands-on training in dental traumatology. Int Endod J. 2018;51(11):1313-9. https://doi. org/10.1111/iej.12947.

36. Sugahara K, Takano M, Koyama Y, et al. Novel condylar repositioning method for 3D-printed models. Maxillofac Plast Reconstr Surg 2018;40(1):04. doi:https://doi.org/10.1186/s40902-018-0143-7.

37. Werz SM, Zeichner SJ, Berg BI, Zeilhofer HF, Thieringer F. 3D printed surgical simulation models as educational tool by maxillofacial surgeons. Eur J Dent Educ. 2018;22(3):e500-5. https://doi.org/10.1111/eje.12332.

38. Lin WJ, Lin W, Lin JC, et al. A novel three-dimensional-printed paranasal sinus-skull base anatomical model. Eur Arch Oto-Rhino-Laryngol. 2018; 275(8):2045-9. https://doi.org/10.1007/s00405-018-5051-z.

39. Haffner M, Quinn A, Hsieh TY, Strong EB, Steele T. Optimization of $3 D$ print material for the recreation of patient-specific temporal Bone models. Ann Otol Rhinol Laryngol. 2018;127(5):338-43. https://doi.org/10.1177/0003489418764987.

40. Jacek B, Radosław W, Wiesław K, et al. 3D printed models in mandibular reconstruction with bony free flaps. J Mater Sci Mater Med. 2018;29(2):10-5. https://doi.org/10.1007/s10856-018-6029-5.

41. Alodadi A. Utilizing three-dimensional printing in treating challenged dental implant cases. World J Dent. 2018;9(3):235-41. https://doi.org/10.5005/jpjournals-10015-1540.

42. Reddy GV, Vasamsetty P, Kumar Malyala S, Alwala A. Training young maxillofacial surgeons or trainees using additive manufacturing. Mater Today Proc. 2018;5(2):4046-9. https://doi.org/10.1016/j.matpr.2017.11.665.

43. Favier V, Zemiti N, Mora OC, Subsol G, Captier G, Lebrun R, Crampette L, Mondaine M, Gilles B. Geometric and mechanical evaluation of 3D-printing materials for skull base anatomical education and endoscopic surgery simulation - A first step to create reliable customized simulators. PLoS One. 2017;12(12):e0189486. https://doi.org/10.1371/journal.pone.0189486.

44. Alrasheed AS, Nguyen LHP, Mongeau L, Funnell WRJ, Tewfik MA. Development and validation of a 3D-printed model of the ostiomeatal complex and frontal sinus for endoscopic sinus surgery training. Int Forum Allergy Rhinol. 2017;7(8):837-41. https://doi.org/10.1002/alr.21960.

45. Wang L, Ye X, Hao Q, et al. Comparison of two three-dimensional printed models of complex intracranial aneurysms for surgical simulation. World Neurosurg. 2017:103:671-9. https://doi.org/10.1016/jwneu.2017.04.098.

46. Javan R, Davidson D, Javan A. Nerves of steel: a low-cost method for 3D printing the cranial nerves. J Digit Imaging. 2017;30(5):576-83. https://doi. org/10.1007/s10278-017-9951-z.

47. Legocki AT, Duffy-Peter A, Scott AR. Benefits and limitations of entry-level 3dimensional printing of maxillofacial skeletal models. JAMA Otolaryngol - Head Neck Surg. 2017;143(4):389-94. https://doi.org/10.1001/jamaoto.2016.3673.

48. Ghizoni E, Ph D, Paulo J, et al. 3D-printed Craniosynostosis model: a new simulation surgical tool. World Neurosurg. 2017. https://doi.org/10.1016/j. wneu.2017.10.025

49. Wiedermann JP, Joshi AS, Jamshidi A, Conchenour C, Preciado D. Utilization of a submental island flap and 3D printed model for skull base reconstruction: infantile giant cranio-cervicofacial teratoma. Int J Pediatr Otorhinolaryngol. 2017;92:143-5. https://doi.org/10.1016/j.jporl.2016.11.014.

50. Jacobo OM, Giachero VE, Hartwig DK, Mantrana GA. Three-dimensional printing modeling: application in maxillofacial and hand fractures and resident training. Eur J Plast Surg. 2018;41(2):137-46. https://doi.org/10.1007/ s00238-017-1373-0.

51. Wanibuchi M, Noshiro S, Sugino T, et al. Training for Skull Base surgery with a colored temporal Bone model created by three-dimensional printing technology. World Neurosurg. 2016;91:66-72. https://doi.org/10.1016/j.wneu. 2016.03.084.

52. Bone TM, Mowry SE. Content validity of temporal Bone models printed via inexpensive methods and materials. Otol Neurotol. 2016;37(8):1183-8. https://doi.org/10.1097/MAO.0000000000001131.

53. Florentino VGB, De Mendonça DS, Bezerra AV, et al. Reconstruction of frontal bone with custom-made prosthesis using rapid prototyping. J Craniofac Surg. 2016;27(4):e354-6. https://doi.org/10.1097/SCS. 0000000000002627

54. Kondo K, Harada N, Masuda $\mathrm{H}$, et al. A neurosurgical simulation of skull base tumors using a 3D printed rapid prototyping model containing mesh structures. Acta Neurochir. 2016;158(6):1213-9. https://doi.org/10.1007/ s00701-016-2781-9.

55. Lim SH, Kim YH, Kim MK, Nam W, Kang SH. Validation of a fibula graft cutting guide for mandibular reconstruction: experiment with rapid prototyping mandible model. Comput Assist Surg. 2016;21(1):9-17. https:// doi.org/10.3109/24699322.2016.1167245.

56. Pacione D, Tanweer $\mathrm{O}$, Berman $\mathrm{P}$, Harter $\mathrm{DH}$. The utility of a multimaterial 3D printed model for surgical planning of complex deformity of the skull base and craniovertebral junction. J Neurosurg. 2016;125(November):1194-7. https://doi.org/10.3171/2015.12.jns151936.

57. Chan HHL, Siewerdsen JH, Vescan A, Daly MJ, Prisman E, Irish JC. 3D rapid prototyping for otolaryngology-head and neck surgery: applications in image-guidance, surgical simulation and patient-specific modeling. PLoS One. 2015;10(9):1-18. https://doi.org/10.1371/journal.pone.0136370.

58. Dickinson KJ, Matsumoto J, Cassivi SD, et al. Individualizing Management of Complex Esophageal Pathology Using Three-Dimensional Printed Models. Ann Thorac Surg. 2015;100(2):692-7. https://doi.org/10.1016/j.athoracsur. 2015.03.115

59. Hochman JB, Rhodes C, Wong D, Kraut J, Pisa J, Unger B. Comparison of cadaveric and isomorphic three-dimensional printed models in temporal bone education. Laryngoscope. 2015;125(10):2353-7. https://doi.org/10. 1002/lary.24919.

60. Cohen J, Reyes SA. Creation of a 3D printed temporal bone model from clinical CT data. Am J Otolaryngol - Head Neck Med Surg. 2015;36(5):61924. https://doi.org/10.1016/j.amjoto.2015.02.012.

61. Rose AS, Webster CE, Harrysson OLA, Formeister EJ, Rawal RB, Iseli CE. Preoperative simulation of pediatric mastoid surgery with 3D-printed temporal bone models. Int J Pediatr Otorhinolaryngol. 2015;79(5):740-4. https://doi. org/10.1016/j.ijporl.2015.03.004.

62. Ernoult C, Bouletreau P, Meyer C, Aubry S, Breton P, Bachelet JT. Reconstruction assistée par l'impression 3D en chirurgie maxillofaciale. Rev Stomatol Chir Maxillofac Chir Orale. 2015;116(2):95-102. https://doi.org/10. 1016/j.revsto.2015.01.010.

63. Mowry SE, Jammal H, Myer C, Solares CA, Weinberger P. A novel temporal bone simulation model using 3D printing techniques. Otol Neurotol. 2015 36(9):1562-5. https://doi.org/10.1097/MAO.0000000000000848.

64. Hochman JB, Rhodes C, Kraut J, Pisa J, Unger B. End user comparison of anatomically matched 3-dimensional printed and virtual haptic temporal Bone simulation: a pilot study. Otolaryngol - Head Neck Surg (United States). 2015;153(2):263-8. https://doi.org/10.1177/0194599815586756.

65. Longfield EA, Brickman TM, Jeyakumar A. 3D printed pediatric temporal Bone. Otol Neurotol. 2015;36(5):793-5. https://doi.org/10.1097/mao. 0000000000000750

66. Rose AS, Kimbell JS, Webster CE, Harrysson OLA, Formeister EJ, Buchman CA. Multi-material 3D models for temporal bone surgical simulation. Ann Otol Rhinol Laryngol. 2015;124(7):528-36. https://doi.org/10.1177/ 0003489415570937.

67. Hochman JB, Kraut J, Kazmerik K, Unger BJ. Generation of a 3D printed temporal bone model with internal fidelity and validation of the mechanical construct. Otolaryngol - Head Neck Surg (United States). 2014;150(3):448-54. https://doi.org/10.1177/0194599813518008.

68. Chenebaux M, Lescanne E, Robier A, Kim S, Bakhos D. Evaluation of a temporal bone prototype by experts in otology. J Laryngol Otol. 2014 128(7):586-90. https://doi.org/10.1017/50022215114001297. 
69. Narayanan V, Narayanan P, Rajagopalan R, et al. Endoscopic skull base training using 3D printed models with pre-existing pathology. Eur Arch Oto-RhinoLaryngology. 2015;272(3):753-7. https://doi.org/10.1007/s00405-014-3300-3.

70. Cui J, Chen L, Guan X, Ye L, Wang H, Liu L. Surgical planning, threedimensional model surgery and preshaped implants in treatment of bilateral craniomaxillofacial post-traumatic deformities. J Oral Maxillofac Surg. 2014;72(6):1138.e1-1138.e14. https://doi.org/10.1016/j.joms.2014.02.023.

71. Gil RS, Roig AM, Obispo CA, Morla A, Pagès CM, Perez JL. Surgical planning and microvascular reconstruction of the mandible with a fibular flap using computer-aided design, rapid prototype modelling, and precontoured titanium reconstruction plates: a prospective study. Br J Oral Maxillofac Surg. 2015;53(1):49-53. https://doi.org/10.1016/j.bjoms.2014.09.015.

72. Jardini AL, Larosa MA, Filho RM, et al. Cranial reconstruction: $3 \mathrm{D}$ biomodel and custom-built implant created using additive manufacturing. J Cranio-Maxillofacial Surg. 2014;42(8):1877-84. https://doi.org/10.1016/j.jcms.2014.07.006.

73. Jabbour $P$, Chalouhi N. Simulation-based neurosurgical training for the presigmoid approach with a physical model. Neurosurgery. 2013;73(SUPPL. 4):2011-4. https://doi.org/10.1227/NEU.0000000000000090.

74. Li J, Li P, Lu H, et al. Digital design and individually fabricated titanium implants for the reconstruction of traumatic zygomatico-orbital defects. J Craniofac Surg. 2013;24(2):363-8. https://doi.org/10.1097/SCS. Ob013e3182701243.

75. Ciocca L, Mazzoni S, Fantini M, Persiani F, Marchetti C, Scotti R. CAD/CAM guided secondary mandibular reconstruction of a discontinuity defect after ablative cancer surgery. J Cranio-Maxillofacial Surg. 2012;40(8):e511-5. https://doi.org/10.1016/j.jcms.2012.03.015.

76. Mori K, Yamamoto T, Nakao Y, Esaki T. Surgical simulation of cerebral revascularization via skull base approaches in the posterior circulation using three-dimensional skull model with artificial brain and blood vessels. Neurol Med Chir (Tokyo). 2011;51(2):93-6 http://www.ncbi.nlm.nih.gov/ pubmed/21358148.

77. Morrison DA, Guy DT, Day RE, Lee GYF. Simultaneous repair of two large cranial defects using rapid prototyping and custom computer-designed titanium plates: a case report. Proc Inst Mech Eng Part H J Eng Med. 2011 ; 225(11):1108-12. https://doi.org/10.1177/0954411911422766.

78. Nikzad S, Azari A, Ghassemzadeh A. Modified flapless dental implant surgery for planning treatment in a maxilla including sinus lift augmentation through use of virtual surgical planning and a 3-dimensional model. J Oral Maxillofac Surg. 2010;68(9):2291-8. https://doi.org/10.1016/j.joms.2010.02.002.

79. Katatny IE, Masood SH, Morsi YS. Evaluation and Validation of the Shape Accuracy of FDM Fabricated Medical Models. Adv Mater Res. 2009;83-86: 275-80. https://doi.org/10.4028/www.scientific.net/amr.83-86.275.

80. Lambrecht JTH, Berndt D, Christensen AM, Zehnder M. Haptic model fabrication for undergraduate and postgraduate teaching. Int J Oral Maxillofac Surg. 2010;39(12):1226-9. https://doi.org/10.1016/j.ijom.2010.07.014.

81. Sohmura T, Kusumoto N, Otani T, Yamada S, Wakabayashi K, Yatani H. CAD/ CAM fabrication and clinical application of surgical template and bone model in oral implant surgery. Clin Oral Implants Res. 2009;20(1):87-93. https://doi.org/10.1111/j.1600-0501.2008.01588.x.

82. Mori K. Dissectable modified three-dimensional temporal bone and whole skull base models for training in skull base approaches. Skull Base. 2009: 19(5):333-43. https://doi.org/10.1055/s-0029-1224862.

83. Radley GJ, Sama A, Watson J, Harris RA. Characterization, quantification, and replication of human sinus bone for surgery simulation phantoms. Proc Inst Mech Eng Part H J Eng Med. 2009;223(7):875-87. https://doi.org/10.1243/ 09544119 JEIM577.

84. Cohen A, Laviv A, Berman P, Nashef R, Abu-Tair J. Mandibular reconstruction using stereolithographic 3-dimensional printing modeling technology. Oral Surg Oral Med Oral Pathol Oral Radiol Endodontol. 2009;108(5):661-6. https://doi.org/10.1016/j.tripleo.2009.05.023.

85. Suzuki M, Hagiwara A, Ogawa Y, Ono H. Rapid-prototyped temporal bone and inner-ear models replicated by adjusting computed tomography thresholds. J Laryngol Otol. 2007;121(11):1025-8. https://doi.org/10.1017/ S0022215107006706

86. Mavili ME, Canter HI, Saglam-Aydinatay B, et al. Use of three-dimensional medical modeling methods for the precise planning of orthognathic surgery. J Craniofacial Surg. 18(4):740-7. https://doi.org/10.4103/JCD.JCD_ 13318.

87. Suzuki M, Ogawa Y, Kawano A, Hagiwara A, Yamaguchi H, Ono H. Rapid prototyping of temporal bone for surgical training and medical education.
Acta Otolaryngol. 2004;124(4):400-2. https://doi.org/10.1080/ 00016480410016478

88. Müller A, Krishnan KG, Uhl E, Mast G. The application of rapid prototyping techniques in cranial reconstruction and preoperative planning in neurosurgery. J Craniofac Surg. 2003;14(6):98. https://doi.org/10.1097/ 00001665-200311000-00014

89. Löppönen $\mathrm{H}$, Holma $\mathrm{T}$, Sorri $\mathrm{M}$, et al. Computed tomography data based rapid prototyping model of the temporal bone before cochlear implant surgery. Acta Oto-Laryngologica, Suppl. 1997;6489(529):47-9. https://doi.org/ 10.3109/00016489709124077

\section{Publisher's Note}

Springer Nature remains neutral with regard to jurisdictional claims in published maps and institutional affiliations.
Ready to submit your research? Choose BMC and benefit from:

- fast, convenient online submission

- thorough peer review by experienced researchers in your field

- rapid publication on acceptance

- support for research data, including large and complex data types

- gold Open Access which fosters wider collaboration and increased citations

- maximum visibility for your research: over $100 \mathrm{M}$ website views per year

At $\mathrm{BMC}$, research is always in progress.

Learn more biomedcentral.com/submissions 